\title{
Integrating Connectivity Into Hydrodynamic Models: An Automated Open-Source Method to Refine an Unstructured Mesh Using Remote Sensing
}

\author{
Kyle Wright $^{1}$, Paola Passalacqua ${ }^{1}$, Marc Simard ${ }^{2}$, Cathleen E. Jones ${ }^{2}$ \\ ${ }^{1}$ Department of Civil, Architectural, and Environmental Engineering, Center for Water and the Environment, University of \\ Texas at Austin, Austin, Texas, USA \\ ${ }^{2}$ Jet Propulsion Laboratory, California Institute of Technology, Pasadena, California, USA
}

\section{Key Points:}

- A method is proposed to integrate remote sensing data into the structure of hydrodynamic model meshes

- We demonstrate this method using optical, InSAR, and topographic data in a model of coastal river deltas

- We observe a one-third reduction in model computational demand in our test application due to the proposed method

- Method is open-source, fully-automated, and agnostic regarding source of remote sensing information 


\section{Abstract}

Hydrodynamic models are an essential tool for studying the movement of water and other materials across the Earth surface. However, the possible questions which models can address remain limited by practical constraints on model size and resolution, particularly in fluvial and coastal environments in which hydrodynamically-relevant landscape features are topologically complex and span a wide range of spatial scales. The rise in popularity of unstructured meshes has helped address this problem by allowing mesh resolution to vary spatially, and many models support local refinement of the mesh using breaklines or internal regions-of-interest. However, there remains no standardized, objective, or easily reproducible method to define or implement internal features between different users. The present study aims to address whether remote sensing information can be used to fill in that gap, by embedding information about hydrological connectivity and landscape structure directly into an unstructured mesh. We present a fully-automated image processing methodology for preserving dynamically-active connected features in the unstructured 2D shallow-water model ANUGA, while reducing computational demand in other less active areas of the domain. The Unstructured Mesh Refinement Method (UMRM) works by converting a binary input raster into a collection of closed, simple polygons which can be used to internally refine the model mesh, meanwhile preserving landscape connectivity and enforcing model-related constraints. The UMRM and ANUGA are both fully open-source and agnostic regarding the source of remote sensing data used as input, which can include optical, radar, and topographic datasets. We demonstrate the use of the UMRM workflow by applying it to a large-scale model of the Wax Lake and Atchafalaya Delta distributary system in coastal Louisiana. Our model mesh is refined using a long-term time-series of optical Planet imagery, a short-term time-series of interferometric SAR measurements of water level change, and lidar-derived topography data. We compare the results of the connectivity-preserving mesh (CPM) to results from an unrefined mesh using a uniform mesh resolution, and find that the UMRM decreased the number of mesh elements, simulation time, and output data size by around a third, without any loss in model accuracy when compared to in-situ and remotely-sensed water level measurements. To our knowledge, this study is the first to use non-topographic remote sensing data to constrain the mesh structure of a hydrodynamic model, and results from our test application suggest that doing so can result in noteworthy reductions in computational demand. 


\section{Introduction}

Due to widespread advancements in computing power and accessibility in the $21 \mathrm{st}$ century, researchers studying the Earth's surface are now able to probe questions regarding the movement of matter and energy through landscapes at unprecedented scales and resolution. The availability of remote sensing imagery and physics-based numerical models have revolutionized the study of large-scale geophysical systems [Balsamo et al., 2018], and enabled simulation of full-scale experiments regarding the effects of different processes on the function and form of landscapes. A number of numerical models have been developed to study the movement of water, sediment, and other materials using finitevolume (FVM) and finite-element (FEM) approaches, and hydrodynamic models in particular have proven to be useful tools for advancing our understanding of hydrological transport and connectivity in fluvial, coastal, and oceanic systems [Lane, 1998; Bates, 2012; Danilov, 2013; Teng et al., 2017; Edmonds et al., 2021]. Hydrodynamic models have been used to study fluvial flooding [Yu and Lane, 2006; Czuba et al., 2019], storm surge [Dietrich et al., 2012; Barbier et al., 2013; Siverd et al., 2019], the transport of biota and nutrients [Arnold et al., 2005; Musner et al., 2014; Hiatt et al., 2018; Große et al., 2019], and have been combined with ecological/morphodynamic models to study landscape change [Fagherazzi et al., 2012; Leonardi et al., 2013; Edmonds et al., 2021; Olliver and Edmonds, 2021], to name only a few applications. Remotely-sensed imagery is often used in conjunction with models, with optical or radar-based measurements used to extract information like inundation extent to aid in model calibration or validation [Horritt, 2000; Schumann et al., 2009]. Hydrodynamic model usage is only likely to increase as software becomes more advanced, accessible, and open-source, and computing power continues to increase through the use of parallelization and cloud computing.

Despite recent advances in computing power and parallelization, the size and complexity of models has remained the primary limit on what can be studied via hydrodynamic modeling. Practical limits exist on the spatial and temporal resolution that can be achieved in models without requiring unreasonably high simulation times or computing power. For an explicit FVM, computational costs, $C$, generally scale with the spatial resolution, $\Delta s$, as $C \propto \Delta s^{-3}$, due to the increasing element count and smaller time-steps required to model a system at higher resolution [Kim et al., 2014]. The same applies for increasing the spatial extent of a model at the same resolution. Even when computing is performed on a computer cluster or in the cloud, model sizes are practically limited by 
time, CPU availability, data storage, and energy usage. Therefore, methods to decrease the computational requirements for a model without sacrificing model accuracy are needed.

Two prevailing frameworks exist for discretizing the landscape into a mesh for use in FVM or FEM models: structured and unstructured meshes [Ferziger et al., 2002]. Structured meshes typically consist of quadrilateral or Cartesian grid-cells of uniform spacing, and have tended to be common in Earth surface modeling because they are relatively simple to implement, they provide the best accuracy in rectilinear channels [Kim et al., 2014], and because their regular grid makes it easy to accurately compute gradients. Models which make use of structured meshes include, for example, Delft-3D [Deltares, 2021a], LISFLOOD-FP [Shaw et al., 2021], and FREHD [Li and Hodges, 2019]. Unstructured meshes, on the other hand, typically consist of triangular or polygonal-shaped elements with variable grid spacing, and have the advantage that resolution can vary spatially and be locally refined around areas of interest. Models which make use of unstructured meshes include ANUGA [Roberts et al., 2015], Delft3D-FM [Deltares, 2021b], MIKE 21 FM [DHI, 2021], and ADCIRC [Luettich et al., 1992]. Some models also make use of a mix of these two approaches, e.g. HEC-RAS 2D [Brunner, 2021], by allowing for some irregular elements or breaklines in an otherwise quadrilateral grid.

When modeling with an unstructured mesh, it is common practice to vary the spatial resolution of the mesh to prioritize resolution within certain regions of interest or in regions with more topographic complexity [e.g. Horritt, 2000; Cobby et al., 2003; Cucco et al., 2009; Schubert and Sanders, 2012; Dietrich et al., 2012; Kim et al., 2014]. Others have proposed a number of novel mesh-generating algorithms to refine the mesh or add breaklines based on elevation or topographic curvature [e.g. Hagen et al., 2001; Cobby et al., 2003; Legrand et al., 2006; Bilskie et al., 2015, 2020; Roberts et al., 2019]. However, most of these methods suffer from a few key drawbacks that limit their application. First, very few of these methods are made open-source and are not readily available for download. Second, many of these methods rely on models/software that are themselves proprietary, such as ADCIRC or Matlab, which further limits their accessibility. And lastly, many of these methods are only "semi-automated," and require user intervention in GIS software to clean or edit the outputs. In fact, for many models, the status quo for implementing breaklines or high-resolution regions-of-interest is entirely decided in a graphical user interface (GUI) based on user judgement (e.g. Delft3D-FM, SMS) - which, as others have pointed out, does not promote objectivity or reproducibility [Roberts et al., 2019]. 
Each of the aforementioned models that utilize an unstructured approach have different methods by which users can adjust the mesh, but in all cases there remains no standardized, reproducible procedure for constructing a model with varying spatial resolution.

In the modern age of big data, the availability of high-quality remote sensing information with which to inform hydrodynamic modeling is unprecedented [Huang et al., 2018; Balsamo et al., 2018]. Topographic lidar, optical imagery, and synthetic aperture radar (SAR) are just a few sensing technologies that have revolutionized Earth-based monitoring over the last few decades. In recent years, it has become increasingly common to use some of these datasets to improve hydrodynamic models. Topographic lidar is now commonly used to improve bathymetric inputs to models [Bates, 2012, 2022]. Optical and SAR imagery, both of which can be used to extract water presence and therefore inundation extent, is now often used as a calibration tool [Horritt et al., 2007; Schumann et al., 2009; Jung et al., 2012; Bates, 2022]. However, these datasets are rarely (if ever) used as constraints on the model mesh itself, and typically only inform the model performance after the "structure" of the model has been fixed, so-to-speak. While most of the aforementioned mesh refinement studies used topographic information to inform model structure, to the best of our knowledge, optical and SAR imagery have never been directly used for this purpose. We argue that this abundance of remotely-sensed information presents an opportunity to make model construction more objective and reproducible, while also reducing computational costs by embedding information about hydrodynamically-relevant landscape features into the model mesh.

The purpose of this study is to introduce a general methodology by which remote sensing imagery of any type can be used to refine the unstructured mesh of the opensource ANUGA hydrodynamic model. Using a set of binary raster images which emphasize hydrodynamic features of interest in the landscape, the Unstructured Mesh Refinement Method (UMRM) uses a few image processing and filtering steps to extract and simplify the regions of interest for a hydrodynamic model. The output of the UMRM workflow is a collection of simple vector polygons that can be used directly as inputs to the built-in ANUGA mesh engine. This method is fully-automated, open-source, and entirely agnostic to the type or source of input data used. To demonstrate the application of this workflow, we apply the UMRM to an ANUGA model of the Wax Lake and Atchafalaya Delta (WLAD) distributary system in coastal Louisiana. We make use of a long-term optical time-series of Planet Labs imagery from the last decade, a short-term 
time-series of water level change derived from interferometric SAR images obtained with UAVSAR, and topographic information from an existing lidar/sonar mosaic to refine our model mesh. We compare the performance of the resulting connectivity-preserving mesh (CPM) ANUGA model with that of an "unrefined" model lacking these remotely-sensed constraints, and discuss changes in computational demand and accuracy that result from applying the UMRM.

\section{Background}

\subsection{Study Site: Wax Lake \& Atchafalaya Deltas}

We illustrate our proposed workflow towards the development of a hydrodynamic model of the Wax Lake and Atchafalaya Delta (WLAD) system in coastal Louisiana (Figure 1). The WLAD system is a frequently modeled landscape [e.g. Liang et al., 2015; Hiatt and Passalacqua, 2017; Xing et al., 2017; Christensen et al., 2020; Olliver and Edmonds, 2021; Shafiei et al., 2021] and exemplifies many of the complex morphological features our method is designed to tackle: channel widths that span a range of scales $O\left(10^{1}-10^{3} \mathrm{~m}\right)$, dendritic and loopy network structures, substantial amounts of channelwetland connectivity [Hiatt and Passalacqua, 2015], and leveed or otherwise hydrologicallyinactive regions adjacent to important flow conduits. While every riverine landscape is ultimately different with unique challenges for designing a model, we think the WLAD application provides a good general example in which to test our methodology.

The morphology of the WLAD distributary system is the result of both natural landbuilding processes and human engineering interventions. This coastal basin includes a majority of the wetlands fed by fluvial water and sediment from the Atchafalaya river, which is a sub-distributary of the Mississippi river and receives about $30 \%$ of its flow annually [Roberts et al., 2003; Allison et al., 2012]. Some of that discharge is then diverted from the Atchafalaya into the Wax Lake Outlet, which is an engineered diversion built by the USACE in 1941 to alleviate flooding in Morgan City [Roberts et al., 2003]. The WLO and lower Atchafalaya distributaries receive an average water discharge of about $2800 \mathrm{~m}^{3} / \mathrm{s}$ and $3600 \mathrm{~m}^{3} / \mathrm{s}$, respectively. The river deltas formed at the mouth of each distributary are actively prograding into Atchafalaya Bay, and have produced about $85 \mathrm{~km}^{2}$ of new land since 1973 [Zhang et al., 2021]. These aggradational delta lobes are the main draw for the numerous modeling studies done in this area, because the Wax Lake Delta has been deemed 


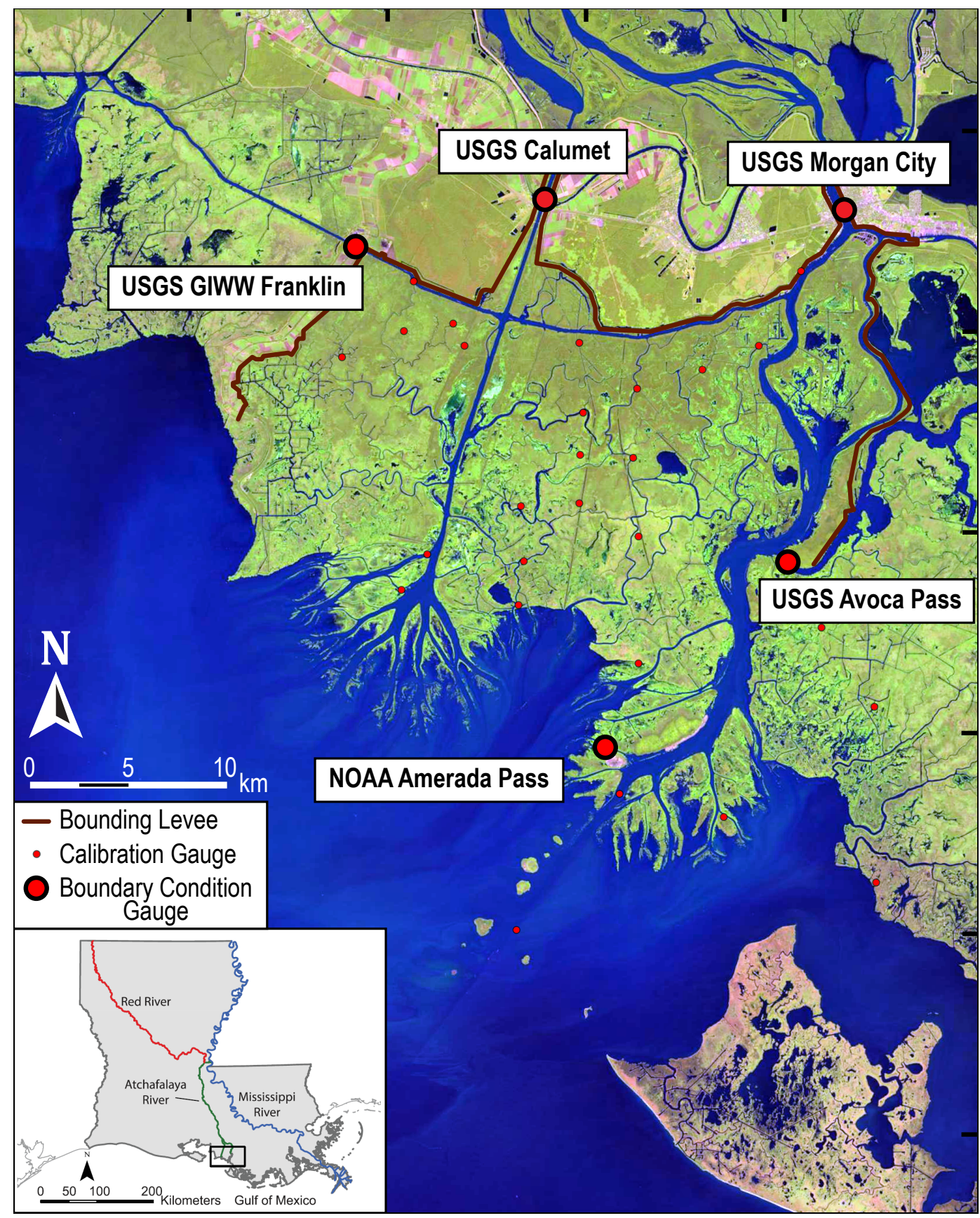

Figure 1. The Wax Lake Delta (left) and Atchafalaya Delta (right) distributary watershed. Permanent water level gauges from CRMS, USGS, and NOAA shown as red circles. Important gauges used to prescribe model boundary conditions at inlets/outlets are highlighted. High-elevation levees bounding the distributary watershed are also shown. False color image taken October 2019, courtesy of LandSat and provided by USGS. 
a natural prototype for the potential of engineered sediment diversions elsewhere in coastal Louisiana [Paola et al., 2011]. Most research has been focused on the Wax Lake Delta itself due to dredging activities in the Atchafalaya Delta - however, the two are functionally connected to each other via an elaborate network of upstream channels and wetlands, and by the Gulf Intracoastal Water-Way (GIWW), which typically flows away from the Atchafalaya in both the East and West directions within this region [Swarzenski, 2003]. For this reason, we choose to model the two subsystems together as one.

Due to research interest in the WLAD system, a number of in-situ and remote sensing datasets were collected to support modeling of this basin. Numerous long-term monitoring gauges and sites exist inside the Atchafalaya distributary watershed (Figure 1), including USGS discharge gauges [USGS, 2016], NOAA tide stations [NOAA, 2016], and Louisiana's own Coastwide Reference Monitoring System (CRMS) stations [LACPRA, 2018]. The area is also the focus of the Pre-Delta-X and on-going NASA Delta-X projects [JPL, 2021], for which a number of remote sensing datasets have been collected, including lidar-derived digital elevation models that have been merged with sonar surveys to produce a high-quality bathymetric dataset [Denbina et al., 2020], and UAVSAR flights to collect synthetic-aperture radar measurements of water level change inside the coastal wetlands [Jones et al., 2021].

\subsection{ANUGA Hydrodynamic Model}

The proposed UMRM is designed to integrate directly with the ANUGA hydrodynamic model [Roberts et al., 2015]. The ANUGA model uses the finite volume method on an unstructured mesh of triangular grid cells to numerically solve the $2 \mathrm{D}$ depth-averaged shallow water equations, which are given as follows:

$$
\frac{\partial}{\partial t}\left(\begin{array}{c}
h \\
u h \\
v h
\end{array}\right)+\frac{\partial}{\partial x}\left(\begin{array}{c}
u h \\
u^{2} h+g h^{2} / 2 \\
u v h
\end{array}\right)+\frac{\partial}{\partial y}\left(\begin{array}{c}
v h \\
v u h \\
v^{2} h+g h^{2} / 2
\end{array}\right)=\left(\begin{array}{c}
0 \\
g h\left(S_{0, x}-S_{f, x}\right) \\
g h\left(S_{0, y}-S_{f, y}\right)
\end{array}\right)
$$

in which $u, v$ are flow velocities in $x, y$ directions, respectively, $h$ is the flow depth, $g$ is gravitational acceleration, $S_{0}$ is the downward bed slope, and $S_{f}$ is the friction slope. The model is coded in Python, with computationally-expensive subroutines written in $\mathrm{C}$ for efficiency, and is fully parallelizable on multiple cores using the Message-Passing Interface (MPI). ANUGA has been used in a number of coastal applications and was tested against a number of analytical test cases, showing good performance [Nielsen et al., 2005; Mungkasi 
and Roberts, 2013; Davies and Roberts, 2015]. ANUGA is fully open-source and freely available on GitHub, which is one of the primary reasons we choose to use it for the current application over other less-accessible proprietary software. Another reason is the simplicity of the mesh engine, which allows users to easily import vector data to change the internal resolution of the mesh. The mesh class contains three methods by which these constraints can be implemented:

- breaklines: An enforced line that mesh cells cannot cross, which helps resolve sharp discontinuities in the topography.

- internal_regions: A closed, simple polygon inside the boundaries of the model with a different mesh resolution than the background value.

- internal_holes: A closed, simple polygon inside the boundaries of the model which is empty (i.e. lacks mesh cells), and can be used to represent urban structures or other impermeable areas.

Each of these methods allows users to carefully optimize the model mesh for their particular application. The ability to quickly import predefined vector data delineating internal polygons allows for direct integration with our proposed methodology. In the present study, we only make use of the internal_regions method - however, potential uses of the other methods are discussed in section 5.3. It is important to emphasize here that the UMRM workflow is designed to operate upstream of the ANUGA mesh engine (and does not re-write it) and its built-in methods, which reduces complexity when trying to apply these methods to different installations or operating systems. Furthermore, it ensures that these methods are not inherently restricted to application with the ANUGA model - any other 2D hydrodynamic software which enables the import of vector data for mesh delineation can make use of the proposed method.

\section{Methods}

Regardless of the choice of input data, the aim of the Unstructured Mesh Refinement Method (UMRM) is to optimize the model mesh to be high resolution in areas that are hydrodynamically-active over the time-scales relevant to the model (e.g. channels, wetlands, lakes) and lower resolution in areas that are hydrodynamically-inactive (e.g. dry land, disconnected wetlands). It is important to mention the caveat here that "active" and "inactive" should be understood as relative terms; this is discussed further in section 5.4. 
We use "hydrodynamically-active" herein to mean "exhibits fluvial or tidal activity under the range of discharges and environmental conditions observed in the imagery and considered by the model." The aim is therefore to use supplementary remote sensing datasets to inform which areas of the landscape fall into each of these categories. In the following sections, we will walk through the process of converting a few remotely-sensed datasets into suitable input layers, merging those layers into a mask of active/inactive regions, cleaning and filtering that mask, enforcing several important constraints for use with a hydrodynamic model, and converting that information into inputs compatible with the ANUGA mesh engine.

\subsection{Data Collection \& Pre-Processing}

We use three remotely-sensed datasets as inputs to our mesh-refinement workflow to inform our model of the WLAD system:

1. High-resolution optical satellite imagery obtained from Planet Labs (both RapidEye and PlanetScope constellations) [Planet, 2018]

2. High-resolution interferometric synthetic-aperture radar (InSAR) airborne imagery from NASA's UAVSAR [Jones et al., 2021]

3. A preexisting lidar/sonar bathymetry mosaic [Denbina et al., 2020]

We collected optical satellite imagery spanning the range from 2009 to 2020 over the WLAD system from Planet Labs [Planet, 2018]. The bounds of the imagery, as with the hydrodynamic model, were chosen to span the extent encompassing the major levee systems on the North, East, and West sides of the distributary watershed (Figure 1) in order to best close the mass balance on the system. For each year in the observation window, we collected the best available 4-band imagery (defined as having the best balance of minimal cloud coverage and the greatest fraction of the system covered) as near as possible to the months identified to be the typical yearly vegetation minimum (Jan-Mar) and maximum (Aug-Oct) [Olliver and Edmonds, 2017], i.e. two downloaded acquisitions per year. In seasons in which no single acquisition provided adequate coverage of the WLAD system, we collected two proximal acquisitions on different dates to fill the gap. We used both RapidEye imagery $(5 m)$ and PlanetScope imagery $(3 m)$, but due to the history of the availability of each of these satellites, a majority of the collected acquisitions were from RapidEye. Each of these satellites provided imagery in the Blue, Green, Red, and NIR 
bands. In total we downloaded 29 acquisitions for use in this study, 22 RapidEye, and 7 PlanetScope. We provide a full list of the precise acquisition dates used in this analysis and their spatial coverage in the supporting information (SI).

For each imagery acquisition date, we merge all tiles together into a single mosaic representing each date in the time-series. We filtered out clouds from each mosaic using the associated Usable Data Mask (UDM) provided by Planet Labs for each image, with UDM2 given priority when available [PlanetLabs, 2018]. Finally, because more RapidEye images were available in the time-series, we used bilinear resampling to rescale the PlanetScope images to match the $5 m$ resolution of the RapidEye images.

We extracted water and vegetation features from each mosaic in the time-series using the well-known Normalized-Difference Water Index (NDWI, Equation 2, McFeeters [1996]) and Normalized-Difference Vegetation Index (NDVI, Equation 3, Rouse et al. [1974]).

$$
\begin{gathered}
N D W I=\frac{\text { Green }-N I R}{\text { Green }+N I R} \\
N D V I=\frac{N I R-\text { Red }}{N I R+\text { Red }}
\end{gathered}
$$

We normalized these values to the range $0-255$ (i.e. 8-bit) using the range of values in each image to correct for differences in environmental conditions between acquisition dates.

To extract open water features, such as channels, lakes, and the bay from these images, we applied Otsu's thresholding method [Otsu, 1979] to each NDWI image in the sequence, which binarizes the image based on the histogram of intensity into water and non-water features. For each pixel in the image, the total number of times the pixel was classified as "water" was normalized by the number of acquisitions over that pixel, thereby representing a temporal average of water presence over the entire system. Finally, we binarized the image into "water" and "non-water" pixels, using a water presence threshold of $C_{1}=15 \%$ to delineate water features. We chose a value of $C_{1}$ to provide a good balance between excluding noise over land pixels and maintaining connectivity between channelized pixels - we discuss the implications of this choice and other constants in section 5.1. This raster of water presence formed the first input layer to the mesh workflow (Figure $2 \mathrm{a}, 3 \mathrm{k})$ in order to ensure that all channels and open-water features are modeled in highresolution. 
In this landscape, inundated wetlands show considerable seasonality in vegetation cover [Olliver and Edmonds, 2017] - inundated herbaceous vegetation tends to sprout in the Spring, peak in the late Summer, and senesce in the Fall/Winter. Likewise, tidal conditions between different acquisitions create variation in the apparent extent of vegetation inside of inundated wetlands. Because of these two features, we argue that high variance in the time-series of NDVI images is a good proxy for the presence of tidally-active inundated wetlands, assuming that variations caused by atmospheric effects are spatially stationary over the imagery extent. From the NDVI time-series, we therefore computed the standard deviation of NDVI for each pixel in the image, and chose a threshold of $C_{2}=40$ which appeared to best delineate known wetlands in the landscape from other more static swamps/marshes. This raster using an NDVI-based proxy for wetland vegetation was the second input layer to the mesh workflow (Figure 2b, 31).

Our second remote sensing dataset consists of six airborne radar acquisitions of the WLAD taken over a span of 2.5 hours by the NASA UAVSAR instrument between 14:08 and 16:37 UTC on October 16th, 2016 as part of the Pre-Delta-X trial campaign [Jones et al., 2021]. UAVSAR uses an active polarimetric L-band synthetic aperture radar, with an incidence angle between 22 and 67 degrees and a $22 \mathrm{~km}$-wide image swath. UAVSAR was flown in a repeat-pass orientation at roughly 30-minute intervals between 14:08 and 16:37 UTC, during which most of the region was experiencing falling tides. These six acquisitions were then used to create five interferograms representing LOS displacement of the water surface between flights. Phase unwrapping was performed using SNAPHU to create maps of water level change during the 2.5-hour observation window [Jones et al., 2021]. Note that UAVSAR does not maintain coherence over open water, so these waterlevel change measurements are only available inside inundated wetlands in which doublebounce scattering off emergent vegetation dominates the return signal. After processing, each of these interferograms had a roughly $7 \mathrm{~m}$ spatial resolution, which we resampled to match the $5 m$ resolution and extent of the RapidEye imagery.

For our third input layer in this analysis, we delineated regions which were tidally active during this 2.5-hour window as any pixels in which the cumulative water level change ever exceeded $C_{3}=3 \mathrm{~cm}$ (Figure $2 \mathrm{c}, 3 \mathrm{~m}$ ). While clouds ostensibly have little effect on SAR imagery, atmospheric distortions are still visible in the resulting interferograms, particularly near the periphery of the images (i.e. near Morgan City and in the Northwest quadrant near Franklin, Figure 2c). However, for the purposes of this application we chose 


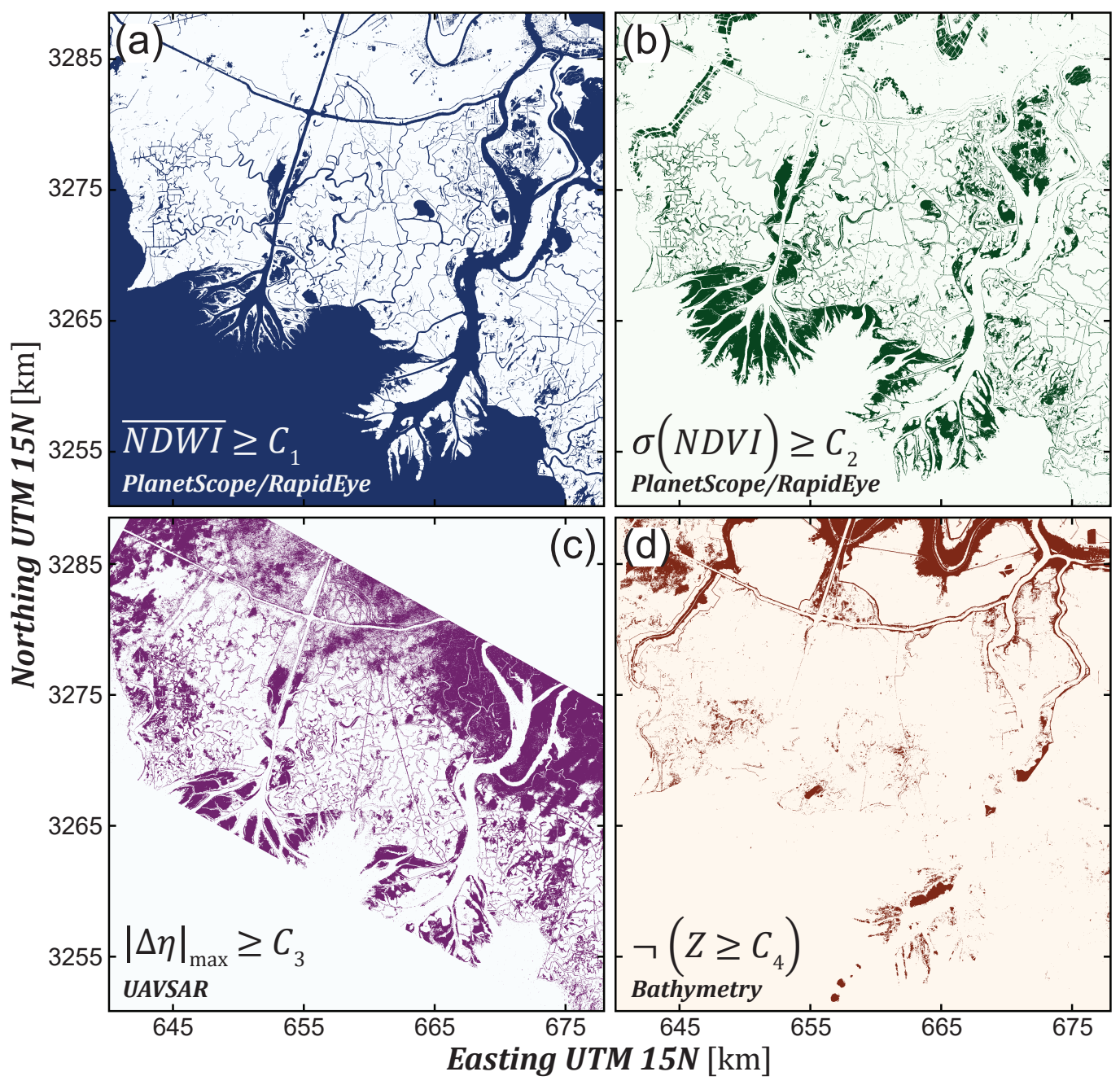

Figure 2. Remote sensing layers used as inputs to the processing workflow to constrain the model mesh. In all layers, the non-white color indicates positive pixels. (a) Long-term water presence extracted from 28 optical RapidEye/PlanetScope images spanning 2009-2020. (b) Herbaceous wetland vegetation used as a proxy for inundated wetlands from the same Planet imagery. (c) Short-term tidal activity extracted from five InSAR maps of water level change from UAVSAR in October 2016. (d) Prohibitively high elevations extracted from the topography mosaic, used as a negative constraint to correct for errors in previous input layers.

to ignore these distortions, because they primarily fell into the category of "false positives" where the extent of tidal activity is larger than reality. Because tidally-active regions are later mapped to high-resolution regions of the model, false positives (resolution greater than what is needed) are preferable to false negatives (resolution less than what is needed) for the purposes of this demonstration. 
We used topography to define our fourth and final input layer [Denbina et al., 2020], which was intended as a negative constraint to correct for some of the noise and errors inherent to the previous layers, in particular the atmospheric noise of the InSAR timeseries. We extracted prohibitively high elevations of the topography using a threshold of $C_{4}=80 \mathrm{~cm}$ NAVD88, which primarily consists of engineered levees and deposits from dredge spoil in the basin (Figure $2 \mathrm{~d}, 3 \mathrm{n}$ ). We chose $C_{4}$ to be an elevation that exceeded local water level measurements but fell below levee elevations. Reinforcing topographic disconnections between nearby water bodies helps to limit the number of aforementioned "false positives" and keep computational demand low. We resampled this topographic layer from its initial $10 m$ resolution to the same $5 \mathrm{~m}$ resolution of the other input layers.

Finally, to merge these four input layers, we took the union of the first three masks (water presence, wetland vegetation, or tidally active), and excluded from it any pixels which were classified in the fourth mask as topographically disconnected. Because the optical time-series and the InSAR time-series capture both long and short timescales of hydrodynamic activity, respectively, our assumption is that the union of both layers represents a relatively unrestrictive definition of "hydrologically active" in the combined raster. The result is a binary image of active and inactive regions which serves as the basis for the UMRM workflow in this case study (Figure 3o).

\subsection{Unstructured Mesh Refinement Method}

\subsubsection{Constraints on Internal Regions}

The UMRM is designed to automate the formatting of a binary raster mask as an input the the ANUGA mesh engine. Streamlining this process requires enforcing a number of constraints on the data before it can be useful with modeling. While some of these constraints are trivial (e.g. data must be stored in vector format), others may be less obvious - some of which are practical (relating to the numerical implementation of the data) and some physical (relating to optimal practices for modeling riverine systems). A few key constraints generally applicable to all ANUGA model domains are as follows:

1. ANUGA requires that all polygons defining internal_regions or internal_holes are closed and simple (polygon boundaries fully enclose a region of space and do not cross each other); in other words, they must be Jordan Curves. 
2. Horizontal spacing between elements in the model should never be forced to be prohibitively small, i.e. smaller than the highest acceptable resolution for a given computational cost. This would cause prohibitively high simulation times according to the CFL stability condition $(u \Delta t / \Delta x) \leq \alpha_{\max }$, where $\alpha_{\max }$ is the maximum Courant number for stability.

3. The boundaries of different polygons must not intersect each other or the boundary - furthermore, there must exist enough space between them such that the mesh cells which fill that space are not prohibitively small.

4. Each polygon vertex will be concentric with triangle vertices in the resulting model mesh. Therefore, polygon vertices must be sufficiently spaced out to avoid prohibitively small triangle elements.

5. Mesh triangles in ANUGA obey a default minimum triangle angle of $28^{\circ}$ for stability. Therefore, angles inside internal polygons should obey the same rule.

6. ANUGA requires internal polygons to be defined in counter-clockwise order.

7. Regardless of the local mesh resolution, the model will be unable to resolve flows to a location if the relevant conduits for those fluxes are blocked elsewhere. Therefore, the UMRM should account for non-local structural connections in the landscape when designating regions as high- or low-resolution.

The filtering and processing steps of the UMRM described in the following sections (Figure $3 p-z)$ take careful measures to address each of these constraints.

\subsubsection{Raster Image Operations \& Filtering}

The first steps of the UMRM (Figure 3p-v) make use of several widely-used and open-source image processing tools in Python, primarily those contained in the imageprocessing package scikit-image [van der Walt et al., 2014], to extract and simplify useful features from the noisy binary input image (Figures 4I, 5a). Descriptions of these processes will be kept at an overview-level, but their effects on the domain are shown in detail at both the local scale (Figure 4) and the global scale (Figure 5), and details on the implementation can be found in the code linked to in the acknowledgements. Throughout this section, "objects" refers to clusters of active pixels (assigned 1), and "holes" refers to clusters of inactive pixels (assigned 0 ). 


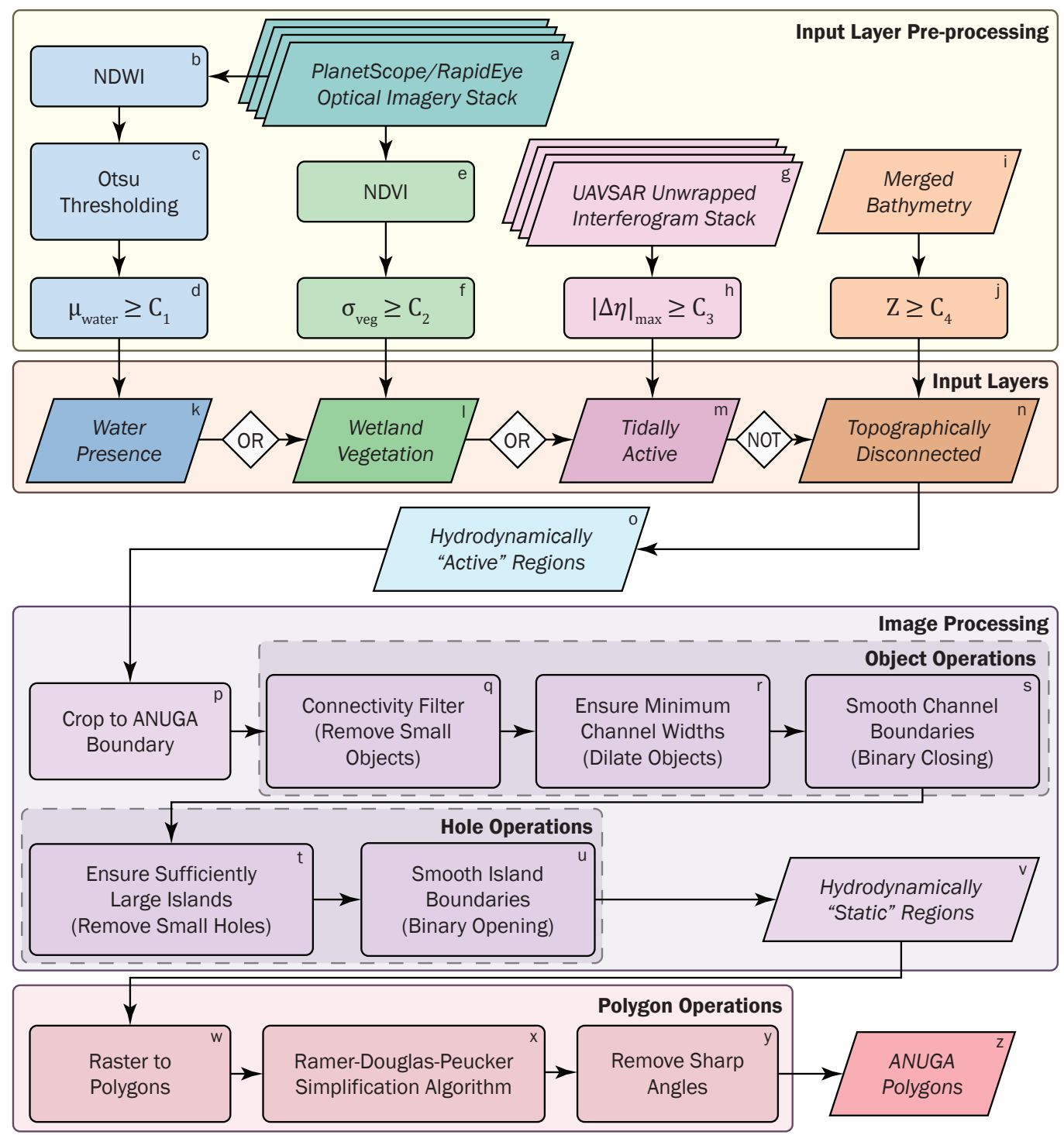

Figure 3. Workflow of the Unstructured Mesh Refinement Method (UMRM)

We begin by first masking out regions of the image which are beyond the extent of the model boundary (Figure 3p). This is the only user-defined structural constraint on the model prior to applying the UMRM. For the WLAD case study, we choose a model boundary that encompasses each major discharge inlet north of the Morgan City and Calumet USGS gauges, and loosely encloses the major levee structures to the North, West, and Northeast of the distributary basin (Figure 1, 2d). Major outlets along the GIWW are included east of the Atchafalaya and west of WLO near Franklin. Lastly, the boundary extends $\approx 30 \mathrm{~km}$ into Atchafalaya Bay. Note that these model boundaries extend outside the extent of the remote sensing data (see section 3.2.4). In order to ensure any resulting in- 
terior_regions do not intersect the model boundary (i.e. Constraint 3), we enforce a $100 \mathrm{~m}$ buffer region between the edges of the mask and the model interior.

Next, we apply a connectivity filter to eliminate any objects which are not connected to the rest of the channel network (Figures 3q, 4ii). This step is related to Constraint 7, and is designed to reduce computational time in regions to which flows are already blocked elsewhere in the channel network, which the model will be unable to resolve regardless of the local resolution. To do this, we compute the area for all objects in the image, and eliminate all but the largest hydrologically-connected cluster.

At this point, it is important to recall Constraint 1 listed in 3.2.1: polygons defining interior_regions must be Jordan Curves. Most coastal channel networks are characteristically 'loopy' and unlikely to satisfy this constraint, as is the case in the WLAD. This challenges what is likely the most intuitive approach for many modelers, which is to selectively increase the model resolution in important regions of the domain. However, the inactive parts of interdistributary islands and marsh platforms are closed and simple by definition, because they are bounded on all sides by the active channel network. Even in less complex landscapes than the WLAD, we expect it to be a common feature of riverine systems that inactive regions are more inclined to satisfy Constraint 1 , due to the simple fact that active regions are presumably hydrologically connected to each other. Therefore, we choose to invert this so-called intuitive approach, and instead define interior_regions in which we selectively coarsen the model resolution within the domain.

In order to ensure that channel levees are captured in high-resolution - and that the boundaries between coarse island regions are not too close in proximity anywhere in the domain - we apply a dilation operator to the largest object cluster using a $50 \mathrm{~m}$ disk (Figures 3r, 4iii). This buffer size was chosen based on the target resolution of the highresolution areas of the model (see section 3.3) to ensure that a minimum of two mesh cells would fit on average between adjacent polygons. This step enforces a minimum channel width throughout the network, and ensures that channel levees will also be captured in high resolution.

As the final object operation, we apply binary closing (dilation followed by erosion) to simplify and smooth the boundaries of the active channel network (Figures 3s, 4iv), utilizing the same buffer size. This process reduces the amount of complexity and noise 
along the interface between the active and inactive regions, and connects nearby active pixels to each other.

We then apply two filtering steps to the holes of the image directly. First, we remove any hole that is too small, inside which the mesh would not be capable of coarsening beyond the background resolution (Figure 3t). We choose a threshold of $0.25 \mathrm{~km}^{2}$ as the threshold size of islands for this operation, based on the target resolutions for the mesh (which will be discussed in section 3.3). Finally, we apply binary opening (erosion followed by dilation, Figure $3 u$ ) to further simplify the boundary of each inactive region and reduce the sharpness of perturbations along the interface created by closing. The end result of these filtering steps is a raster image in which the remaining gaps between the active channel/wetland network (Figures $4 \mathrm{v}, 5 \mathrm{~b}$ ) represent areas in which the model mesh could be suitably coarsened without sacrificing model accuracy in active areas.

\subsubsection{Vector Operations}

Vector polygons are then extracted from the raster image and stored as a list of $(x, y)$ vertices for each inactive region (Figure $3 \mathrm{w}$ ). After this operation, the default number of vertices defining each polygon are typically $1-2$ orders of magnitude greater than what is desired, and would pose challenges in the model relating to Constraint 4 . We apply the Ramer-Douglas-Peucker (RDP) algorithm to decimate the vertex count and simplify each polygon to their essential shape [Douglas and Peucker, 1973] using an $\varepsilon=50 \mathrm{~m}$. This process tends to reduce the number of vertices to $O(10-100)$ points (Figure $3 \mathrm{x}$ ).

In order to enforce Constraint 5, we compute the angle $\theta$ between each subsequent pair of polygon vertices, and eliminate any vertices with $\theta \leq 28^{\circ}$ or $\geq 332^{\circ}$ (Figures $3 y$, 4vi). This step ensures that mesh triangles are not forced to fill in these thin segments with acute triangles, which would lead to stability issues and disobey the ANUGA minimum angle constraint.

Finally, for each polygon, we loop through each list of vertices in counter-clockwise order and save them to disk as a regular text file. During this process, we also delete the redundant last vertex of the polygon (which is concentric with the first) to avoid supplying overlapping vertices to the ANUGA mesh engine. This is the final step of the UMRM (Figure $3 z, 5 c$ ), and the result is a list of text files defining coarse interior_regions satisfying each constraint listed in 3.2.1 that can be directly imported into ANUGA. 

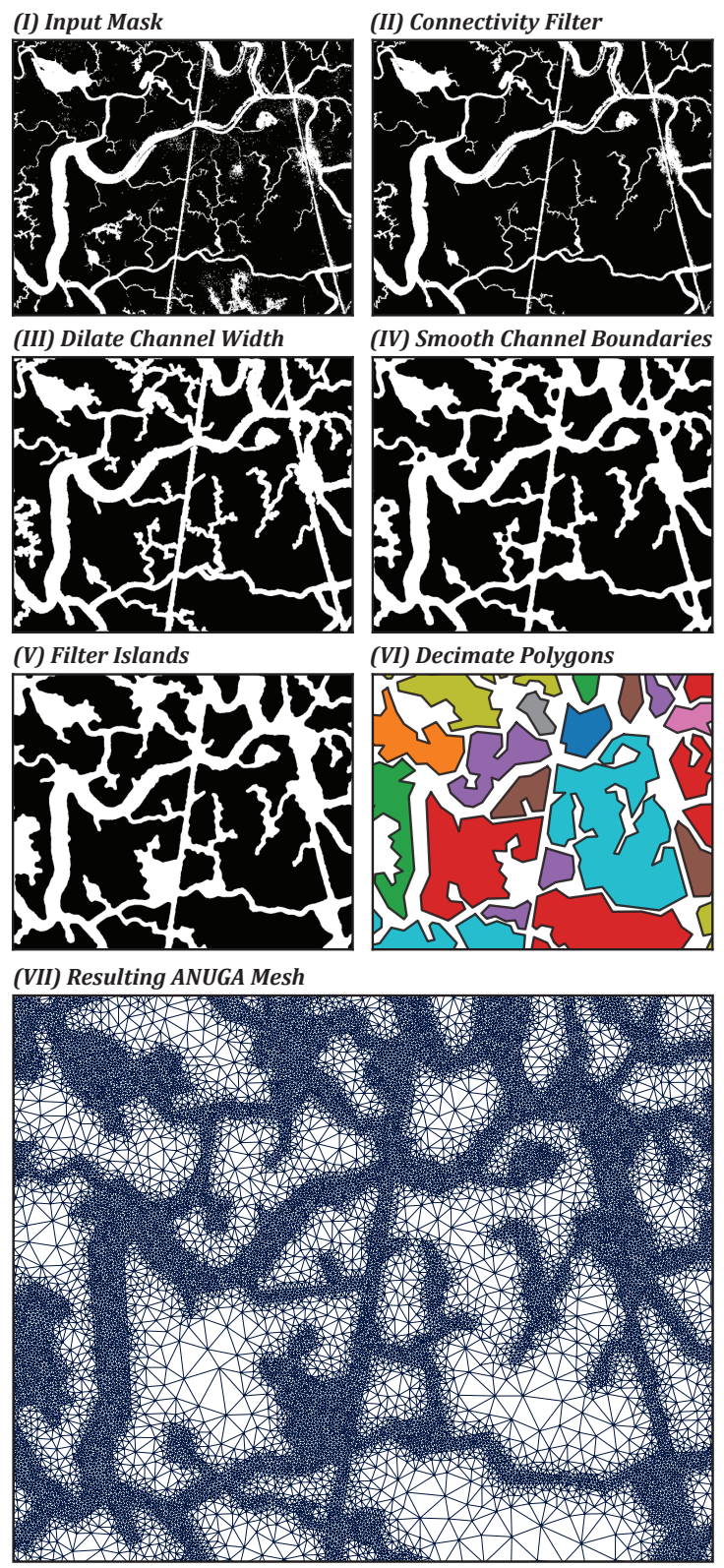

Figure 4. Main steps of the mesh refinement method, with the local effects of each processing stage shown in detail for a small sub-region of the WLAD domain. (I-VI) Filtering steps simplifying the mask of active regions into closed, simple polygons, between which network connectivity is preserved in high-resolution. White indicates hydrodynamically "active" cells. (VII) ANUGA mesh for this sub-region based on the resulting polygons. The location of this inset is shown in Figure 5a

\subsubsection{Additional Processing Outside Imagery Extent}

As was noted in 3.2.2, the extent of the model boundary is greater than that of the input imagery of our region of interest in the WLAD. This boundary was chosen to safe- 

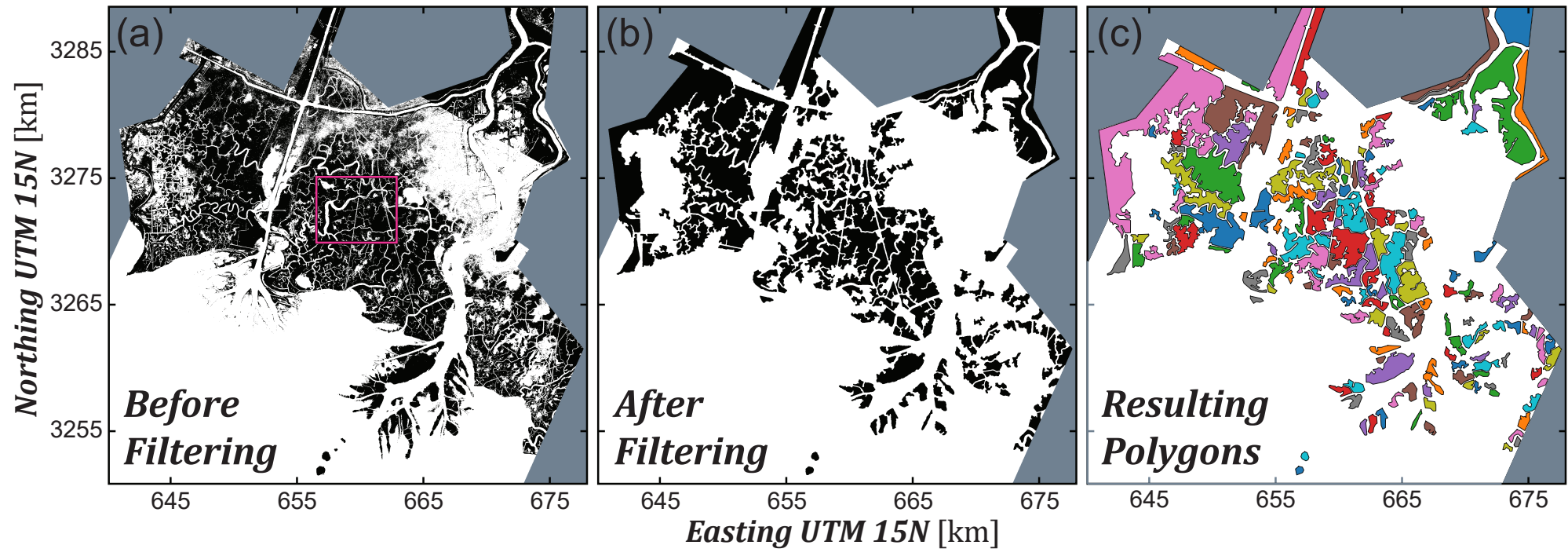

Figure 5. Main steps of the mesh refinement method, with the global effects of select processing stages shown over the full WLAD domain. (a) Unfiltered input mask delineating hydrodynamically "active" (white) and "inactive" regions (black), the result of merging the input layers in Figure 2. (b) Raster of active and inactive regions after application of the raster-based filtering steps. (c) Vector form of the inactive region polygons after applying the polygon-based processing steps.

guard the model results from numerical effects near the tidal boundary, while also keeping the data size of the input layers manageable. As a result of this choice, the model mesh is significantly higher resolution out in the open-water bay than is needed or desirable. While these areas are still hydrologically "active" in the sense used in earlier sections, coarsening the mesh far away from the coast where topographic gradients are very low is common practice in ocean modeling [e.g. Hagen et al., 2001; Bilskie et al., 2020].

In order to further reduce the model simulation time, we define a few additional polygons outside of the imagery extent using select steps of the UMRM and comparatively simple input criteria. Using the topography/bathymetry raster as our only input, we threshold the raster into "land" (=0) and "non-land" (=1) pixels using a (conservative) threshold of $-1 m$ NAVD88. We then apply binary closing (Figure $3 u$ ) using a window size of $300 \mathrm{~m}$, which was determined by trial-and-error to be large enough to close a majority of the inland channels of the WLAD. Remaining channel segments are eliminated by removing small objects (Figure 3q), leaving only three remaining regions in the image: open water in Atchafalaya Bay, Marsh Island (Southeast corner of Figure 1 which 
we choose to exclude from our region of interest and model in coarse resolution), and upstream inland wetlands (which are within the imagery extent and do not need additional refinement). Note that, in this instance, the operations in $\mathrm{u}$ and $\mathrm{q}$ of the UMRM are applied as pre-processing, in order to convert the raw topography raster into a useful input mask

We extract the pixels assigned to Atchafalaya Bay and Marsh Island into different independent rasters, and then to each raster object we mask out the model boundary (Figure 3n), erode each by a buffer of $100 m$ (inverse of Figure 3r) to enforce some distance between them, and apply all polygon operations of the UMRM (Figure 3w-y). Due to their simple topology (and the smooth boundary already attained via pre-processing), none of the intermediate steps of the UMRM are necessary. The result is two additional input polygons to coarsen the bay. We apply all of these same operations to the two hightopography areas surrounding each discharge inlet North of the imagery extent to produce two more (smaller) supplementary polygons. While these four additional polygons are attained using most of the same logic and operations as those described in sections 3.2.23.2.3, because they are not derived using the same input imagery layers, we consider them to be outside of the main scope of the WLAD model demonstration. Therefore, both the refined mesh model and the control model described in the following section make use of these supplementary polygons. We do this to emphasize the model performance inside the interior wetland channels, and to help keep the control model computationally tractable. A brief discussion on the influence of the supplementary polygons in particular is provided in section 5.2.

\subsection{Test Model Setup}

We construct two ANUGA models of the WLAD to demonstrate the functionality of the UMRM. The first model, which we label the connectivity-preserving mesh (CPM), makes use of the interior_regions defined in section 3 to coarsen select regions of the domain. The second, which we label the unrefined mesh, uses a uniform grid resolution everywhere equal to that of the high-resolution areas of the CPM. Both models make use of the supplementary polygons outside the imagery extent (from section 3.2.4), so they only differ in the inclusion of polygons in the interior wetlands (i.e. those shown in Figure 5c). 
We simulate each model to match the environmental conditions of October 15th18th 2016 to align with data collected as part of the Pre-Delta-X campaign. Discharge inflows from upstream in the WLO and Atchafalaya are set to equal the average discharge over the simulation window as measured at the Calumet (\#07381590) and Morgan City (\#07381600) USGS gauges, which equates to $1645 \mathrm{~m}^{3} / \mathrm{s}$ and $2144 \mathrm{~m}^{3} / \mathrm{s}$, respectively. Two smaller discharge outlets are also enforced along the GIWW. The first, at the Western outlet, is set to match the average flow rate of $-106 \mathrm{~m}^{3} / \mathrm{s}$ measured at the USGS gauge near Franklin (\#07381670). The second, at the Eastern outlet, lacked a discharge gauge, and is instead forced using the average flow rate measured at USGS ADCP transects near the Avoca Pass gauge (\#073816501), or $-140 \mathrm{~m}^{3} / \mathrm{s}$. All discharge inflows/outflows are enforced in ANUGA using an inlet_operator, which is recommended to ensure the correct mass flow rate into the system. Around each inlet/outlet, we slightly modify the topography to create a shallow pool, in order to help reduce the reflection of tidal harmonics off the boundary and back into the domain, and to provide a buffer region in which flows can stabilize before entering the domain. Tides are enforced using a time-varying Dirichlet boundary set to equal the water level time-series measured at the NOAA Amerada Pass gauge (\#8764227). The tidal time-series is shifted $\Delta t=100$ minutes earlier in time in order to correct for the position of the model boundary relative to the gauge, where $\Delta t$ is computed by comparing the cross-covariance between the measured and modeled water levels at the calibration gauges. All other model boundaries are set as no-flux (i.e. reflective) boundaries.

Friction in the model is prescribed using a classification map containing six friction classes: (1) bay, (2) large channels, (3) small channels, (4) subtidal vegetation, (5) intertidal vegetation, and (6) supratidal vegetation (see SI for map details and coefficients). For all of these classes, the friction term $S_{f}$ in Equation 1 is parameterized using the Chézy equation:

$$
S_{f, i}=\frac{u_{i} \sqrt{u^{2}+v^{2}}}{C_{z}^{2} h} \quad i \in\{x, y\}
$$

in which $C_{z}$ is the Chézy coefficient. For friction classes (1-3), which are all open-water, $C_{z}$ is parameterized according to Manning's equation:

$$
C_{z}=\frac{h^{1 / 6}}{n}
$$


where $n$ is the Manning's coefficient for each roughness class. For friction classes (4-6), which are all vegetated, $C_{z}$ is parameterized according to the Baptist equation:

$$
C_{z}=\sqrt{\frac{1}{\left(1 / C_{b}^{2}\right)+\left(C_{D} m D h_{v} / 2 g\right)}}+\frac{\sqrt{g}}{K} \ln \left(\frac{h}{h_{v}}\right)
$$

in which $C_{b}$ is the Chézy coefficient of the bed $(\approx 65), C_{D}$ is the drag coefficient, $m$ is the vegetation stem density, $D$ is the stem diameter, $h_{v}$ is the stem height (which only comes into play for non-emergent vegetation), and $\kappa \approx 0.4$ is von Karman's constant. In each vegetation class, $m, D$, and $h_{v}$ are initialized to match typical values found in the WLAD system, and $n$ is initialized using typical values from the literature. While the other parameters are held constant, $n$ and $m$ are adjusted via trial-and-error during the calibration process. We use the built-in ANUGA implementation of Manning's equation for classes (13), and a custom user-defined baptist_operator implementation of the Baptist equation for classes (4-6).

We utilize three mesh resolutions in the model, prescribed as the max allowable triangle area within that region of the domain:

- $625 \mathrm{~m}^{2}$ - High-resolution regions of the domain in both the CPM and unrefined models, chosen to yield $\mathrm{a} \approx 25 \mathrm{~m}$ grid spacing between mesh elements as an acceptable balance between resolving channel features and computational demand.

- $62,500 \mathrm{~m}^{2}$ - Coarse-resolution regions of the domain in the CPM model (absent from the unrefined model), chosen to yield $\mathrm{a} \approx 250 \mathrm{~m}$ grid spacing between mesh elements inside the UMRM-defined internal_regions (Figure 5c)

- $1 \mathrm{~km}^{2}$ - Lowest-resolution regions out in the bay in both the CPM and unrefined models, implemented inside the Atchafalaya Bay and Marsh Island supplementary polygons defined in section 3.2.4

Note that these resolutions represent maximum cell sizes prescribed to the ANUGA mesh engine - the mean actual cell size will therefore be smaller than these ceiling values, according to local characteristics of the mesh and/or polygons (e.g. proximity to a border). The spatial variability in actual cell sizes can be seen in the resulting mesh (Figures 4 and 6 a show the CPM mesh - the unrefined model mesh is comparatively trivial and not shown). The resulting sizes of each mesh are 1,544, 332 cells in the CPM model and 2, 222, 138 cells in the unrefined model, with similar minimum $\left(106 \mathrm{~m}^{2}\right.$ and $\left.104 \mathrm{~m}^{2}\right)$ and maximum $\left(0.995 \mathrm{~km}^{2}\right.$ and $\left.0.997 \mathrm{~km}^{2}\right)$ cell sizes for each model (respectively). These mesh 
cells are then populated with topographic information (Figure 6b) using the preexisting bathymetry mosaic [Denbina et al., 2020], after applying corrections to fix a few locations of erroneous hydro-flattening in some of the interior wetland channels (details in the SI).

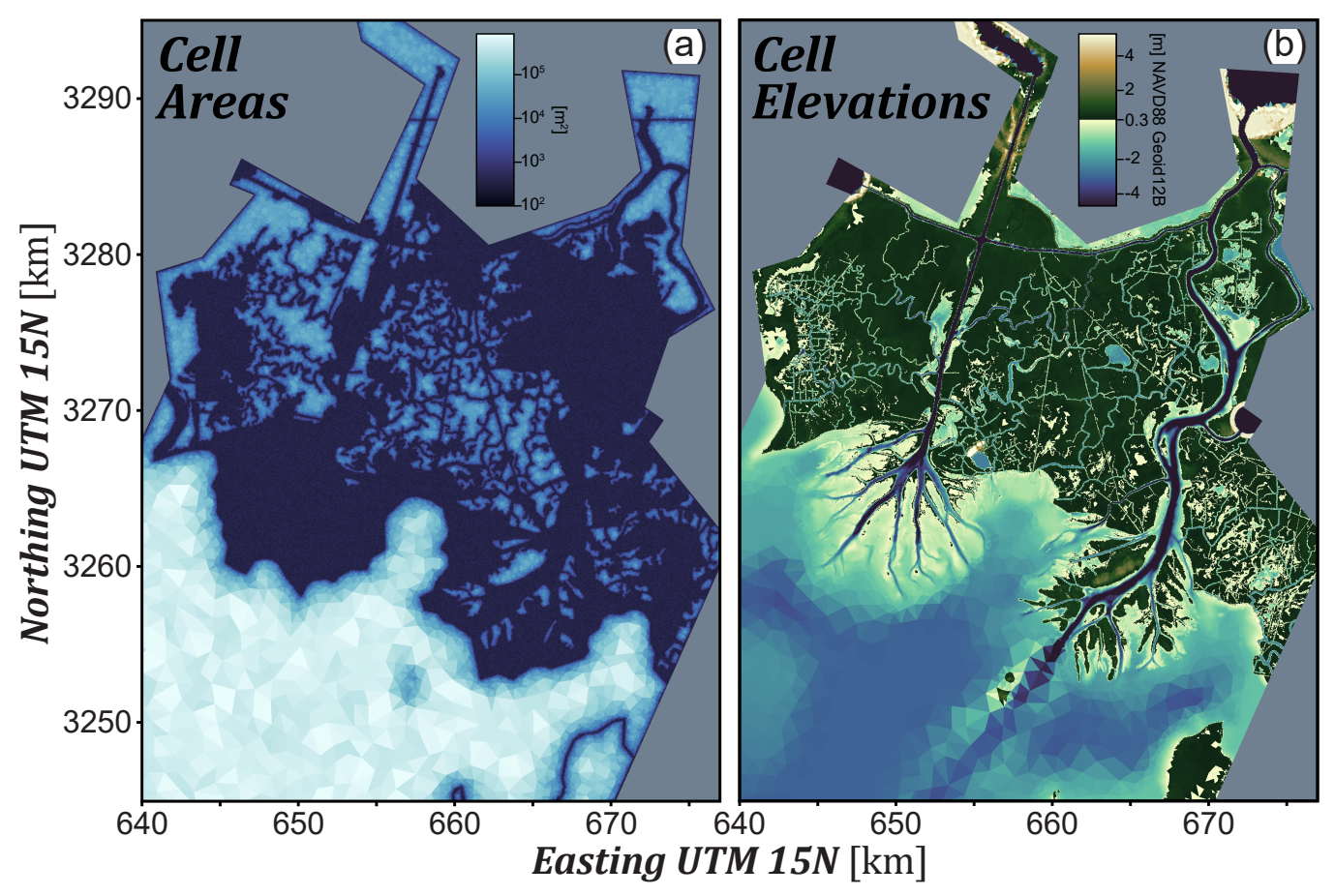

Figure 6. Connectivity-preserving ANUGA model domain after application of the UMRM (a) Connectivitypreserving mesh (CPM) in which cells are colored by their respective area, which is low resolution inside the interior_regions specified in Figure 5c (b) CPM model topography, in which cells are colored by their topographic elevation. The color discontinuity is set to $0.3 m$ NAVD88, which is approximately mean-high water in the WLD

Model simulations are performed in parallel on the Stampede2 cluster of the Texas Advanced Computing Center (TACC). Each simulation is distributed between 8 Intel Xeon Skylake nodes and 48 tasks per node (384 tasks total). To provide the greatest accuracy in the low-Froude landscape of the WLAD, all simulations use the ANUGA "DE1" flow algorithm and the low_froude setting to reduce flux-damping. Each model is run for four days (model time) to allow tidal flows to stabilize prior to the simulation window. Timesteps in ANUGA are variable and internally-optimized based on the CFL condition, but the model yieldstep (the interval at which model outputs are saved to disk) was chosen to be every 15 minutes. 
To evaluate the performance of each model, we compare modeled water levels to those measured at the 30 permanent gauge stations which had available data for the simulation window (3 NOAA, 6 USGS, and 21 CRMS), as well as at 10 additional temporary gauges installed for the Pre-Delta-X campaign [Simard et al., 2020]. Water level measurement frequencies varied by source agency, and were 5-minute (JPL), 6-minute (NOAA), 15-minute (USGS), and 1-hour (CRMS) respectively. All gauge measurement times were converted to UTM, and all water level measurements were referenced to the NAVD88 datum using Geoid12B. Two USGS gauges and one NOAA gauge lacked a NAVD88 reference datum, and two NOAA gauges lacked a reference geoid, and a systematic vertical bias may exist for these gauges. If a gauge completely lacked a reference datum, its measurements were offset to match the mean elevation of the nearest gauge with a verified datum, which is a fair assumption given the shallow slopes $\approx O\left(10^{-5}\right)$ in this system. In addition, we evaluate simulations with data collected over several lidar flights conducted during the simulation window and processed to extract the water surface elevations in the WLO [Denbina et al., 2021]. We compare error statistics - such as the Root-Mean-Square Error (RMSE), Mean Absolute Error (MAE), the coefficient of determination $\left(R^{2}\right)$, and the mean vertical offset $\left(\mu_{\Delta \eta}\right)$ - between the CPM and unrefined models. We also qualitatively compare the InSAR-derived water level change measurements to the simulated rate of water level change to guide our choice of vegetation density $m$ in the trial-and-error calibration process.

It should be noted that the aim of the current study is not to develop a perfectly calibrated model of the WLAD system - with the quantity of calibration data available, it is likely one could apply a more sophisticated approach to fine-tune the friction parameters used in this model implementation. Rather, the aim of the present study is to show the change in model performance due to changes in the mesh as a result of applying the UMRM, while all other attributes of the model (e.g. friction, boundary conditions) are held constant. The model calibration performed herein was deemed more than sufficiently accurate (based on error statistics) for the purpose of model comparison, but calibration itself is not the focus of this study.

\section{Results}

It is clear from the resulting sizes of the CPM and unrefined meshes $(1,544,332$ and 2,222, 138 cells, respectively) that applying the UMRM successfully reduced the to- 
tal number of elements in the CPM by nearly a third (30.5\%) compared to the unrefined mesh. The reduction in simulation times is similar, with the CPM model taking on average $31.5 \%$ less clock time than the unrefined model to finish an identical simulation - in total a reduction from approximately $12.9 \mathrm{hr}$ to $8.9 \mathrm{hr}$. Both models had similar time-step statistics, with an average time-step of $0.213 s$ and $0.204 s$ in the CPM and unrefined models, respectively.

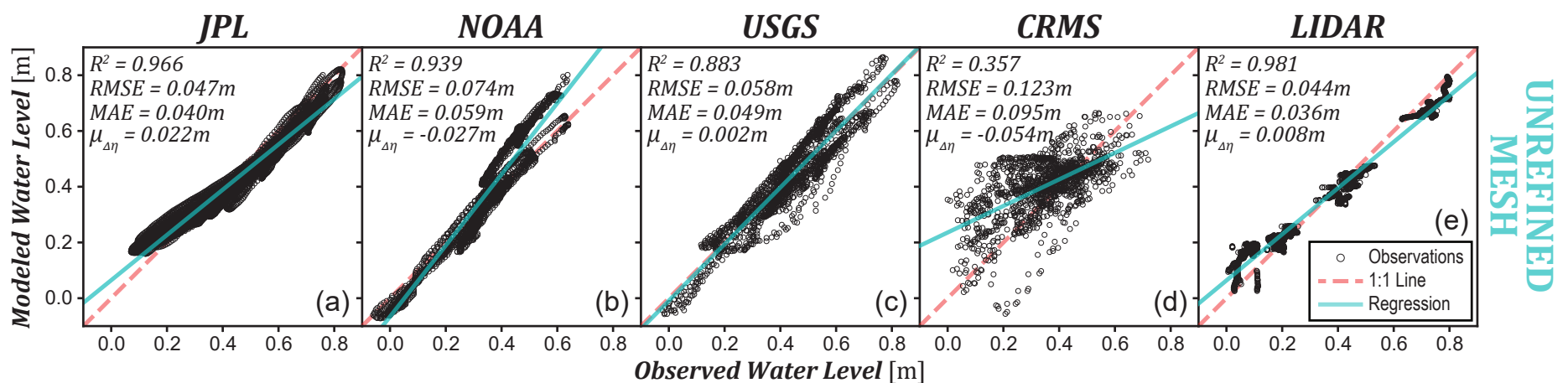

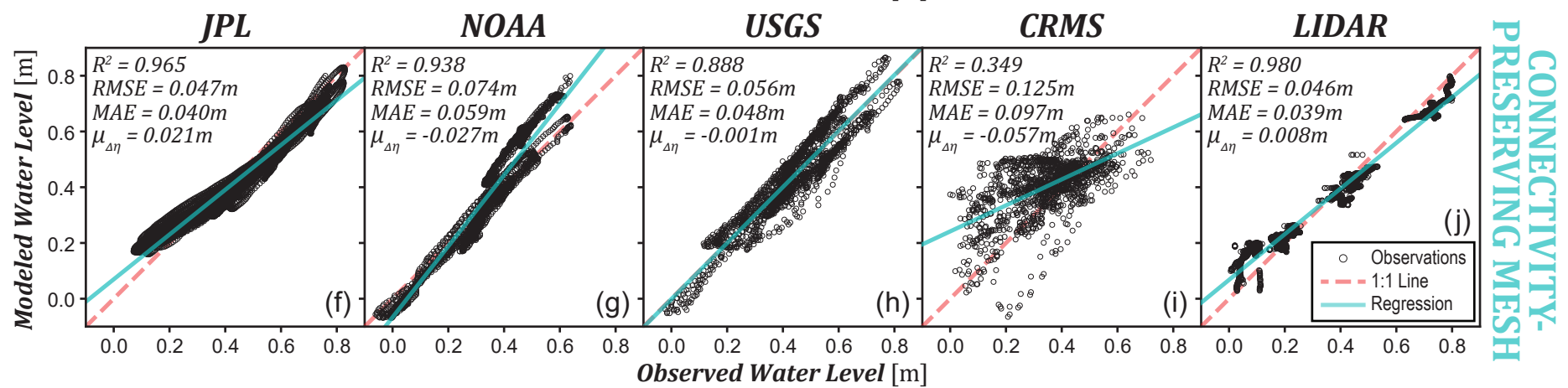

Figure 7. Performance of each model compared to water level measurements collected during the Pre-

Delta-X campaign, sorted by data source for the (a-e) unrefined mesh and (f-j) connectivity-preserving mesh.

For each data series, we indicate each water level measurement, the 1:1 perfect-prediction line and linear regression, and error statistics. The CPM and unrefined mesh have nearly identical performance at reproducing measured water levels.

Model performance statistics were very similar between the CPM and unrefined models (Figure 7). Both models performed relatively well at reproducing measured water levels at a majority of gauges in the region, as well as the lidar-derived water levels. In general, both models performed better at gauges near larger channels or water bodies (e.g. the WLO, Atchafalaya, GIWW) than in smaller channels in the interior of the wetlands. This is reflected in the poorer performance at CRMS gauges (Figure 7d,i) located deeper 
into wetlands along small and shallow channels. This is due to the well-known numerical diffusion of momentum across channel banks [Davies and Roberts, 2015], which increases channel drag, particularly in places where the highest mesh resolution $(\approx 25 \mathrm{~m}$ grid spacing $)$ in both models is larger than the smallest $(\approx 10 \mathrm{~m})$ channels. Despite this shortcoming, tidal propagation is still visible in most of those channels in a diminished form, and it does not appear to affect the performance of the other gauges in larger channels.

Approximately four CRMS gauges $(0301,4779,4808,4809)$ did not show any appreciable tidal activity in either model, with water levels remaining approximately static throughout the simulation window. The latter three gauges are all clustered together in the interior wetlands southwest of the GIWW-WLO intersection, whereas 0301 is southeast of the GIWW-Atchafalaya intersection. We estimate from Google Earth that the mean channel size associated with these four CRMS gauges is approximately $11.2 \mathrm{~m}$. All four of these gauges were flagged as active by the input mask (Figure 5a), and only one of the four was reclassified as inactive during the UMRM - 4779 was removed by the connectivity filter (Figure 3q) due to a disconnection upstream. Even though three of these locations were modeled with high resolution by the CPM model - and all of them by the unrefined model - neither model was well-suited to observe tidal activity at these gauges, which implies that the UMRM is not primarily responsible for poor performance at these four gauges.

All performance statistics were nearly identical between the CPM and unrefined models - they did, however, vary by source agency. Model RMSE varied between 4.4 $12.3 \mathrm{~cm}$ depending on the data source, with a mean of about $6 \mathrm{~cm}$ across all measurements. MAE was generally lower, ranging from $3.9-9.7 \mathrm{~cm}$ with a mean of about $5 \mathrm{~cm}$. Both error measures were generally lowest for lidar-derived data (Figure 7e,i) and highest for CRMS data (Figure 7d,i). For the NOAA and USGS gauges, these error metrics may be artificially inflated slightly due to uncertainty in the reference datum of a few of the gauges, as mentioned in section 3.3. Despite this, the USGS linear regression has near-perfect agreement with the 1:1 observed-modeled line for both models (Figure 7c,h). Non-CRMS data all have generally good $R^{2}$ values and show good clustering around the 1:1 line. Several of the regressions demonstrate a slope $<1$, which suggests that the current calibration may be slightly under-predicting the tidal range on average. However, the NOAA and USGS gauges observed the largest tidal range of any of the source agencies, and do not show the same bias in the regression slope. 
The scale and pattern of water level change in both simulations are comparable to the InSAR-derived measurements of water level change during the 2.5 hour observation window on October 16th, between 14:08 and 16:37 UTC (Figure 8). This observation window coincided with the turning of low tides, with the tidal minimum occurring at about 15:30 at the coastline, as indicated by the NOAA Amerada Pass gauge on the western edge of the Atchafalaya Delta (Figure 1). The tidal response in the interior wetlands is delayed with respect to the coastline due to the finite propagation speed of the wave front - as a result, InSAR primarily measured the falling limb of the tidal signal, with the rising limb only visible in the most distal reaches of the WLAD, such as inside the Pintail Bar and Johnston Islands of the WLD (Figure 8a). The InSAR-derived spatial patterns and direction of water level change inside large wetlands are reasonably well captured by both the CPM (Figure 8b,e,h) and unrefined (Figure 8c,f,i) models, with large interior wetlands lowering several centimeters within the window, and then beginning to rise again in the most distal islands.

The large wetlands inside the interdistributary islands of each delta complex (Figure $8 \mathrm{a}, \mathrm{d})$ tend to show the best qualitative agreement with the InSAR measurements. However, some differences can be seen between the magnitudes of the measured and simulated water level change in a few locations. In a few wetlands, water levels appear to have fallen too much or too little, with more visible differences in the Atchafalaya Delta (Figure 8d-f) than in the WLD (Figure 8a-c). We hypothesize that this is reflective of the fidelity of the bathymetry data in each delta used when constructing the topographic mosaic [Denbina et al., 2020] - intertidal bathymetry in the WLD was sourced from more recent and carefully-constructed datasets [Shaw et al., 2016], whereas data quality in the Atchafalaya is more uncertain. The largest differences in the measured and simulated water level change, however, occurs in the upstream more interior wetlands. North of the WLD, for example, several small channels are visibly associated with falling water levels in the InSAR data (Figure 8a), but neither model succeeds in capturing these dynamics (Figure $8 \mathrm{~b}-\mathrm{c}$ ). While water does tend to inundate these locations during high tide, no water level change was visible during the low-tide InSAR observation window. The fact that both the CPM and unrefined models failed to observe any low-tide activity in those locations suggests this is an overarching effect of the grid resolution, rather than anything relating to the UMRM. In fact, it is clear from the locations of interior polygons (Figure 

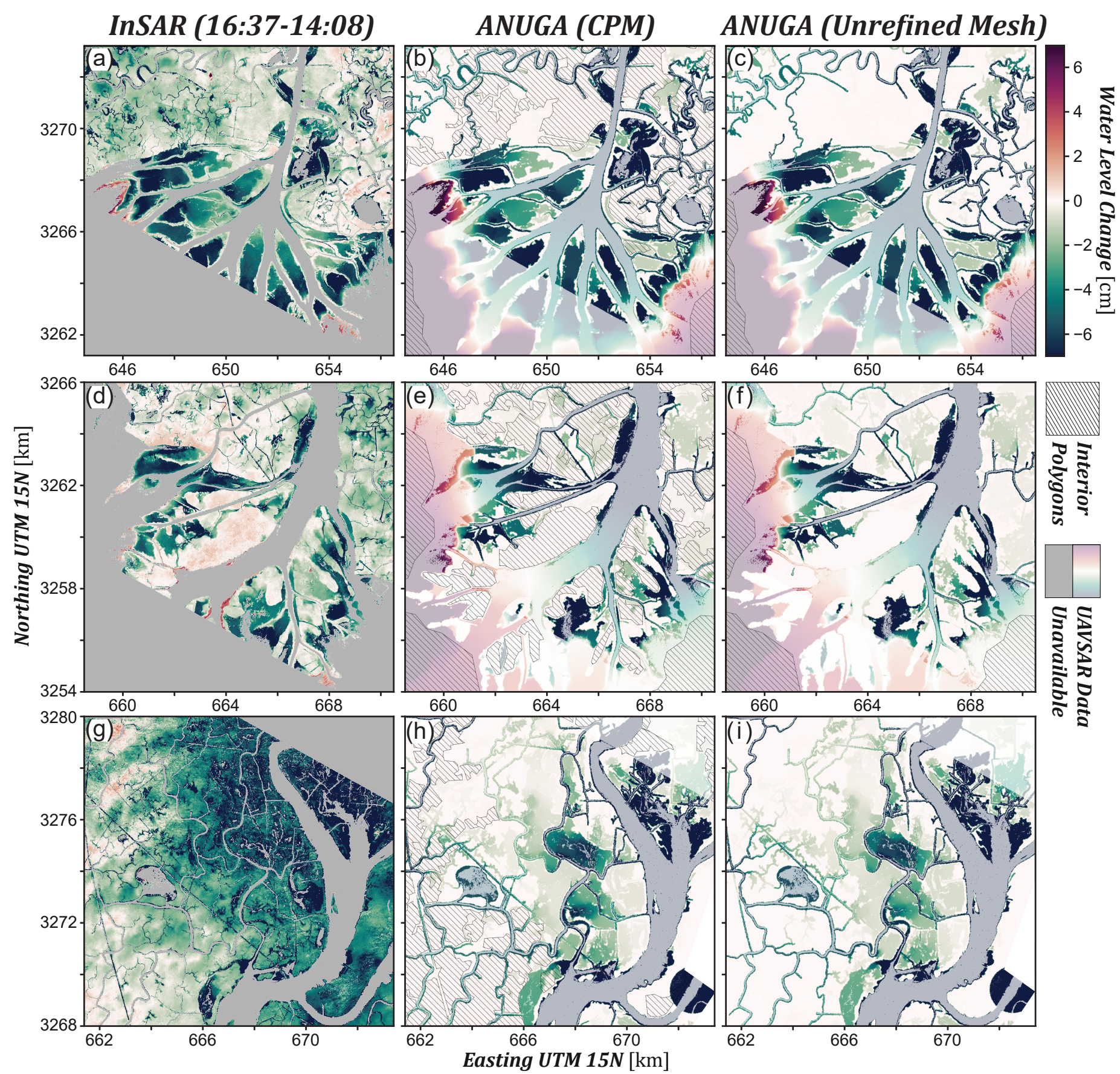

702 Figure 8. InSAR-derived water level change compared to simulated water level change for the connectivity-

703 preserving mesh (CPM) and unrefined mesh models in three noteworthy sub-regions of the domain: (a-c) the

704 Wax Lake Delta, (d-f) the Atchafalaya Delta, and (g-i) the upstream wetlands of the Atchafalaya. Open-water

705 locations which lack interferometry data are shown in either grey (for the data) or as a semi-transparent mask

706 (for the models). Locations of coarsened interior regions are indicated with a hatch overlay. 
8b) that the UMRM tried to preserve connectivity in these locations, which were classified as active by all three (positive) input masks (Figure 2a-c).

Disagreements between the simulations and InSAR data are greater in the upstream wetlands along the Atchafalaya (Figure 8g-i), with the models failing to resolve falling water levels in numerous interior wetlands. We hypothesize that the cause of this discrepancy is two-fold. First, there are several signs that the InSAR-derived water level decreases in this region are exacerbated by atmospheric distortions, leading to an artificial background signal of falling water levels. Intermediate interferograms within this window show diagonal striping over these locations [Jones et al., 2021], which is a common sign of clouds in the troposphere. This is also suggested by the $3-6 \mathrm{~cm}$ measured decrease in water levels in the island to the northeast of the USGS Avoca Pass gauge and GIWW, which is known to be a high elevation region (Figure 2d) and contains a storm surge levee (Figure 1) that the USACE maintains at an elevation of $\approx 3.5 \mathrm{~m}$ NAVD88. It is highly improbable that this location would have been submerged at the range of discharge and tidal levels measured during the observation window, so $3-6 \mathrm{~cm}$ is likely a good approximation for the excess atmospheric distortion in this region. The second cause of the simulation discrepancy is the quantity of sub-grid-scale natural and artificial channels present in this region of the landscape. The area has numerous canals with widths $<25 m$ throughout, so the mesh discretization appears to have disconnected several noteworthy lakes from the rest of the active channel network. Wetlands which do appear qualitatively similar to the InSAR measurements all drain through sufficiently large channels to be captured by the model resolution.

In general, we did not identify any obvious instances in which the coarsening of the CPM caused by the UMRM was responsible for decreases in the accuracy of the CPM when compared to the unrefined model. The only hydrodynamic differences which could be discerned at all were slight changes in the patterns of inundation in a few locations during high tide, which in the CPM tended to be somewhat more smooth (due to the coarsening of the mesh) than in the unrefined mesh - however, these flooding patterns generally did not differ in magnitude or extent. Most of the inaccuracies in either model were the result of other constraints placed on the model, such as the maximum mesh resolution or input bathymetric quality. 


\section{Discussion}

\subsection{Role of Input Data on Mesh Characteristics}

It is interesting to compare the resulting inactive-region mask (Figure 5b) to the input layers used (Figure 2) and the unprocessed imagery of the WLAD (Figure 1) to observe which regions of the channel network are inactive enough to get classified as such. For example, nearly the entirety of the natural WLD was classified as active and modeled in high-resolution, whereas the numerous anthropogenic dredge spoil deposits in the Atchafalaya Delta are too high-elevation to be tidally active. Many of the upstream interior locations which were delineated as inactive are known to be forested [Thomas et al., 2019] and appear to be a different color in the (false-color) LandSat imagery (Figure 1) which may reflect a relationship between fluvial activity and vegetation reflectance characteristics in the WLAD. Regardless, many of the locations which are clearly active, particularly in wetlands with emergent vegetation, may appear to be less active when viewed as a single snapshot in time (e.g. Figure 1). This highlights the importance of choosing representative input data when determining where to prioritize computational resources.

Due to the importance of channels in conveying flow, it is appropriate that the connectivity filter (Figures 3q, 4ii) is the most restrictive step of the UMRM in deciding which locations get prioritized. Close inspection of the mesh and resulting topography (Figures 4vii, 6) demonstrate that preserving channel connectivity remains a priority all the way through the UMRM to the final mesh. For this reason the water mask (Figure 2a) is the most significant layer to include as an input because it enforces that the resulting polygons obey the channel network structure of the landscape. When other lowerresolution inputs are used to define this water mask in other applications, it is important to ensure that the resolution does not artificially disconnect the network in sub-grid-scale channels, if those channels are expected to convey a hydrodynamically significant flux. Using a longer temporal average of water presence, a lower NDWI threshold, or a filter to reconnect water features to each other (e.g. dilation, binary closing) could help ensure that the network structure is representative of on-the-ground landscape features.

Each of the first three input masks (Figure 2a-c) had some mix of unique and redundant information when compared to the other masks. The wetland vegetation and InSAR input masks (Figure 2b-c) each had the effect of expanding the extent of active pixels delineated in the water mask (Figure 2a), particularly in the areas surrounding wetland 
boundaries and channel banks. It is somewhat surprising how much the vegetation and InSAR layers had in common with each other - the most notable discrepancy between them being the large regions of activity visible in the InSAR mask on the Northern edge of the swath, wherein the true signal of falling tides appears to have been exacerbated by artificial noise caused by moisture in the troposphere. Because both of these input layers emphasize intertidal wetlands, which others have shown (particularly inside the Wax Lake Delta) to have an important hydrodynamic influence on hydrological connectivity and system function [e.g. Hiatt and Passalacqua, 2015; Hiatt et al., 2018; Olliver and Edmonds, 2021], each of these input layers helps ensure that high-resolution channel-island hydrodynamics are maintained in the CPM. The ability of InSAR to see through vegetation does provide unique information in certain locations, particularly in small channels west of the WLO, around which much more activity is visible in the InSAR-derived mask than in either optically-derived mask. Because the InSAR mask uses a different sensing technique, has a different time-scale of observation, and is processed entirely independently of the optical water mask, we believe this to be the next most important input layer (after the water mask) at ensuring that the resulting mesh is well-suited to model a diverse set of locations and conditions. In general, we recommend that future applications of the UMRM use multiple lines of independent observations to ensure that the resulting mesh is not limited by shortcomings inherent to one particular sensor or sensing technique, unless there exists strong confidence in the quality of one particular dataset. Regardless, these results suggest that NDVI variance may be a useful proxy for where to expect hydrodynamic activity in future InSAR missions.

In the case of all input masks, our aim was to use the most conservative choice of binarization thresholds $C_{1}-C_{4}$ applicable to our domain. However, we do expect the exact efficiency improvements of the UMRM in the WLAD to be sensitive to any choice of threshold. In the case of the channel network, for example, choosing too high of a $C_{1}$ is likely to disconnect smaller channels in the network, and choosing too low a $C_{1}$ is likely to mislabel artificial noise as an active water body. In general, the latter of these two options is preferable, because false positives tend to get filtered out by the processing steps of the UMRM. Our choices of thresholds were based on field experience and comparisons with other land classification datasets [e.g. Carle et al., 2014; Olliver and Edmonds, 2017; Thomas et al., 2019; Marshak et al., 2020], and our results suggest that these choices were in fact conservative, given the large swaths of the CPM model in which no activity was 
observed despite being located in a high-resolution region. We expect that as automatic feature extraction software continues to improve [e.g. Isikdogan et al., 2017; Jin et al., 2021], this workflow will become less dependent on any particular choice of threshold.

\subsection{Effects of the UMRM on Performance}

As is clear from the results in section 4, application of the UMRM caused a noteworthy increase in the efficiency of the CPM model simulation without any substantial loss in simulation quality when compared to the unrefined model. The CPM model managed to achieve the same performance with $\mathrm{a} \approx 30 \%$ decrease in element count, simulation time, and resulting output file size by prioritizing computational time and resources in areas that are more hydrodynamically active. We observed only minor changes in the error statistics of water level measurements (Figure 7) and qualitatively similar patterns of water level change (Figure 8) between both models. The fact that simulation efficiency could be improved by a third without any loss in performance suggests that application of the UMRM in this system achieved these efficiency improvements "for free," so-to-speak, without requiring a new mesh algorithm, sophisticated changes to the calibration, or proprietary software.

It is important to note that large swaths of the unrefined model were still coarsened out in Atchafalaya Bay (section 3.2.4) in order to keep the computational demands of the unrefined model within reasonable bounds. While these supplementary regions are not based on the imagery datasets and are therefore not the main focus of the mesh comparison, these additional polygons were still obtained via steps of the UMRM - therefore, the $30 \%$ reduction in computational demand we observe could be considered conservative.

The computational gain resulting from the application of the UMRM depends on the complexity of the landscape. Application of the UMRM in other systems would likely differ in the precise quantity of efficiency improvements that could be obtained through mesh refinement, which would directly depend on the fraction of the model domain that could be reasonably classified as "active" and "inactive". In fully-inundated or other wellconnected settings, it is likely that the UMRM would not offer significant efficiency improvements. However, in many large-scale complex systems containing regions of fluvial/tidal inactivity or fully leveed islands (e.g. the Ganges-Brahmaputra-Meghna Delta, Jarriel et al. [2020]), the potential efficiency increases from applying the UMRM could be 
substantial. Perhaps most importantly, the UMRM could assist in making feasible some large-scale models that might otherwise be intractable. Rather than lowering resolution or narrowing the model bounds, which may limit the kinds of science questions a model would be able to answer, the UMRM could be a new tool in the toolbox to reduce the computational demand required to model a system.

\subsection{Regions, Breaklines, or Holes?}

In the WLAD case-study, inactive interior polygons were enforced in the model using the interior_regions method, inside of which the mesh was coarser than the background but still fine enough to allow for flooding of the marsh platform. However, as mentioned in section 2.2, two other built-in methods for refining the mesh exist which could have been used instead - internal_holes and breaklines. While these were less appropriate for the present application, they could certainly be useful or even preferable in other systems. We will therefore briefly discuss how these other implementations would change our results.

If the internal regions of the WLAD had been enforced as internal_holes, it would have further reduced the computational demand by eliminating all cells inside inactive polygons. This could be a reasonable assumption in many other coastal settings containing flood control structures or embankments, with regions entirely disconnected from fluvial or tidal processes. Other studies have used internal_holes to play the role of buildings and other structures in smaller-scale models in urban settings [e.g. Schubert and Sanders, 2012], which could also be delineated using the UMRM if high-enough resolution data (e.g. UAV imagery) were available. However, inaccurately assigning locations that actually should have flooded to internal_holes (due e.g. to noisy or incorrect input data) would have the effect of overly-confining flows to the channel and leading to unphysical behavior. In the WLAD CPM, a few locations designated as inactive still showed signs of activity in the model simulation (Figure 8e), which would not have been allowed had we used internal_holes. In summary, using internal_holes instead of internal_regions could lead to additional efficiency improvements, but caution should be given as to when the assumption of complete inactivity is appropriate.

If the internal regions of the WLAD had been enforced as breaklines, it likely would have decreased the efficiency of the CPM model when compared to the unrefined model. 
This is because breaklines would increase the complexity of the mesh over the background case without coarsening any areas to compensate, which would ultimately lead to more mesh elements and longer simulation times. However, breaklines could improve the model performance inside the channel network if they properly aligned with channel boundaries, due to better representation of the channel planform. If improved model performance is a higher priority than reduced computational demand, this could be desirable, particularly in smaller models. For implementations of the UMRM using breaklines, we recommend reducing the dilation buffer size (Figure 3r) to keep polygon boundaries better aligned with channels, and reducing the minimum island size threshold (Figure 3t) to retain more of the topographic discontinuities.

\subsection{Other Limitations}

The quality of the output of the UMRM is necessarily limited by the quality, quantity, and resolution of data used as an input to this workflow. The aim of our proposed refinement method is to use remotely-sensed supplementary information to improve numerical models "upstream" of calibration, by embedding topological attributes of the landscape into the structure of the mesh itself. Naturally, this approach is only recommended if that remote sensing information is believed to be representative of on-the-ground conditions in the landscape. Over-confidence in the quality of a small amount of input data could lead to poor performance in the resulting model, much in the same way that over-calibration to unrepresentative calibration data can lead to an unphysical model.

Perhaps the most important caveat regarding the UMRM is embedded in the definition of what it means to be hydrodynamically "inactive". A region labeled inactive under certain environmental conditions could certainly become active during fluvial flooding or storm surge, and could certainly still be a storage space for groundwater/rainfall and serve an important ecological function. It is important to recognize that the choice to refine certain regions of the model domain at the expense of other regions is a value judgement about which kinds of physical processes are most important for the model to capture. In many applications, we believe this is an acceptable tradeoff, but it may not be appropriate in all systems or at all times. In the WLAD, for example, most of the locations labeled "inactive" in the present model would be flooded during the high-discharge season in the Spring. We aimed to be conservative with our inactivity assumption by using input data spanning a long observation window (optical Planet data spanning from 2009 to 
2020), but calibration and validation were still performed exclusively using data during the low-flow month of October. Note that this is not a limitation unique to applications of the UMRM - models are always designed, calibrated, and validated with certain environmental conditions in mind, outside of which performance may be less reliable. The same rules apply when choosing how to (or whether to) refine the mesh.

\subsection{Reproducibility}

Some of the central advantages of the UMRM are that the process is open-source, entirely automated, and reproducible. For a given input mask, the method only requires that the user choose the size of the buffer to use between regions (here $50 \mathrm{~m}$ ), the minimum size of islands to convert into polygons (here $0.25 \mathrm{~km}^{2}$ ), and the $\varepsilon$ used for the RDP algorithm (here $50 m$ ). In principle, this approach could help make the model development more simple, straightforward, and objective. Many models which allow for variable mesh resolution have no automated implementation method, and rely heavily on user judgement when choosing where to place polygons/breaklines, which has the potential to bias model results. The UMRM takes a majority of this process out of the user's hands, and can provide significant increases in model efficiency without requiring a lot of complex decisionmaking directly by the user. Furthermore, existing mesh algorithms that do attempt to refine the mesh based on landscape characteristics typically only account for topography, which under-utilizes other informative types of remotely-sensed data that are becoming more readily available.

The UMRM is entirely agnostic regarding the type of input mask used to delineate active and inactive regions, which modelers could construct using the best available data in the region of their model domain. Because the UMRM and ANUGA are both opensource, this means that different users with access to the same input mask (and details regarding the settings used) can construct the same model from scratch on their own machine, following the same workflow. This could potentially aid in making hydrodynamic models more reproducible, even in instances when the model itself may be restricted from sharing or is otherwise inaccessible. 


\section{Conclusions}

To the best of our knowledge, this study is the first to present a generalized method to use non-topographic remote sensing data to constrain the mesh structure of a hydrodynamic model. The Unstructured Mesh Refinement Method (UMRM) is open-source, fullyautomated, and entirely agnostic regarding the source of imagery data used as input. The method requires only a binary raster and a few parameter choices as inputs, and using a few image processing and filtering steps, produces as output a set of internal polygons for selectively coarsening the mesh. All UMRM outputs are designed by default for numerical stability and compatibility with the mesh engine of the ANUGA hydrodynamic model. Because this workflow and the ANUGA model are both open-source, the availability of this tool can potentially aid in making the process of model development more straightforward, objective, and reproducible. Our test application of the UMRM to a large-scale model of the Wax Lake and Atchafalaya Delta system led to a roughly 30\% decrease in the number of mesh cells, the simulation time, and the resulting output size of the data, without any discernible loss in model accuracy. We hope future studies will quantify the impact of the UMRM on models of other systems and using other types of remote sensing imagery as inputs. We recommend that future applications carefully consider whether the type of remote sensing data used and the assumptions that went into processing those layers are compatible with the science questions being addressed by the model. In addition, we recommend that future applications be conservative regarding which areas of the domain are hydrodynamically active under the environmental conditions being considered. Future work will investigate the possibility of integrating the UMRM with other unstructured 2D hydrodynamic models, as well as with other novel topography-based mesh-generating algorithms. In summary, the reduction in model computational demand demonstrated herein for the WLAD model can serve as motivation for additional usage of remote sensing imagery to inform hydrodynamic model structure in other applications.

\section{Acknowledgments}

This work was supported by the NASA Delta-X project, which is funded by the Science Mission Directorate's Earth Science Division through the Earth Venture Suborbital-3 Program NNH17ZDA001N-EVS3. UAVSAR data courtesy NASA/JPL-Caltech. This work was carried out in part at the Jet Propulsion Laboratory, California Institute of Technology, under contract with the National Aeronautics and Space Agency. The ANUGA model 
and simulation outputs from this study are available for download at deltax.jpl.nasa. gov/data/download/ and the final versions will be accessible via the ORNL DAAC. The Unstructured Mesh Refinement Method codes are open-source and available at github. com/passaH20/meshrefinement. We welcome contributions to the UMRM and encourage anyone to open an issue or pull request on GitHub to offer suggestions or improvements. We thank the developers of ANUGA, particularly Stephen Roberts and Gareth Davies, who have always provided very helpful feedback regarding issues with ANUGA. The ANUGA model is available for download at github.com/GeoscienceAustralia/anuga core. We also thank the rest of the Delta-X research team.

\section{References}

Allison, M. A., C. R. Demas, B. A. Ebersole, B. A. Kleiss, C. D. Little, E. A. Meselhe, N. J. Powell, T. C. Pratt, and B. M. Vosburg (2012), A water and sediment budget for the lower Mississippi-Atchafalaya River in flood years 2008-2010: implications for sediment discharge to the oceans and coastal restoration in Louisiana, Journal of Hydrology, 432, 84-97, doi:10.1016/j.jhydrol.2012.02.020.

Arnold, W. S., G. L. Hitchcock, M. E. Frischer, R. Wanninkhof, and Y. Peter Sheng (2005), Dispersal of an introduced larval cohort in a coastal lagoon, Limnology and Oceanography, 50(2), 587-597, doi:10.4319/10.2005.50.2.0587.

Balsamo, G., A. Agusti-Panareda, C. Albergel, G. Arduini, A. Beljaars, J. Bidlot, E. Blyth, N. Bousserez, S. Boussetta, A. Brown, et al. (2018), Satellite and in situ observations for advancing global Earth surface modelling: A Review, Remote Sensing, 10(12), 2038, doi:10.3390/rs10122038.

Barbier, E. B., I. Y. Georgiou, B. Enchelmeyer, and D. J. Reed (2013), The value of wetlands in protecting southeast Louisiana from hurricane storm surges, PloS one, 8(3), e58,715, doi:10.1371/journal.pone.0058715.

Bates, P. D. (2012), Integrating remote sensing data with flood inundation models: how far have we got?, Hydrological processes, 26(16), 2515-2521, doi:10.1002/hyp.9374.

Bates, P. D. (2022), Flood inundation prediction, Annual Review of Fluid Mechanics, 54, doi:10.1146/annurev-fluid-030121-113138.

Bilskie, M. V., D. Coggin, S. C. Hagen, and S. C. Medeiros (2015), Terrain-driven unstructured mesh development through semi-automatic vertical feature extraction, $A d$ vances in Water Resources, 86, 102-118, doi:10.1016/j.advwatres.2015.09.020. 
Bilskie, M. V., S. C. Hagen, and S. C. Medeiros (2020), Unstructured finite element mesh decimation for real-time hurricane storm surge forecasting, Coastal Engineering, 156, 103,622, doi:10.1016/j.coastaleng.2019.103622.

Brunner, G. (2021), HEC-RAS 2D User's Manual, version 6.0, US Army Corps of Engineers.

Carle, M. V., L. Wang, and C. E. Sasser (2014), Mapping freshwater marsh species distributions using WorldView-2 high-resolution multispectral satellite imagery, International journal of remote sensing, 35(13), 4698-4716, doi:10.1080/01431161.2014.919685.

Christensen, A., R. R. Twilley, C. S. Willson, and E. Castañeda-Moya (2020), Simulating hydrological connectivity and water age within a coastal deltaic floodplain of the mississippi river delta, Estuarine, Coastal and Shelf Science, 245, 106,995, doi: 10.1016/j.ecss.2020.106995.

Cobby, D. M., D. C. Mason, M. S. Horritt, and P. D. Bates (2003), Two-dimensional hydraulic flood modelling using a finite-element mesh decomposed according to vegetation and topographic features derived from airborne scanning laser altimetry, Hydrological processes, 17(10), 1979-2000, doi:10.1002/hyp.1201.

Cucco, A., G. Umgiesser, C. Ferrarin, A. Perilli, D. M. Canu, and C. Solidoro (2009), Eulerian and lagrangian transport time scales of a tidal active coastal basin, Ecological Modelling, 220(7), 913-922, doi:10.1016/j.ecolmodel.2009.01.008.

Czuba, J. A., S. R. David, D. A. Edmonds, and A. S. Ward (2019), Dynamics of surfacewater connectivity in a low-gradient meandering river floodplain, Water Resources Research, 55(3), 1849-1870, doi:10.1029/2018WR023527.

Danilov, S. (2013), Ocean modeling on unstructured meshes, Ocean Modelling, 69, 195210, doi:10.1016/j.ocemod.2013.05.005.

Davies, G., and S. Roberts (2015), Open source flood simulation with a 2D discontinuouselevation hydrodynamic model, Proceedings of MODSIM 2015.

Deltares (2021a), Delft3D-FLOW, Simulation of multi-dimensional hydrodynamic flows and transport phenomena, including sediments, User Manual, version 3.15, Deltares Delft, The Netherlands.

Deltares (2021b), Delft3D-FM, D-FLOW Flexible Mesh, User Manual, version 2022.02, Deltares Delft, The Netherlands.

Denbina, M., M. Simard, T. Pavelsky, A. Christensen, K. Liu, and C. Lyon (2020), PreDelta-X: Channel Bathymetry of the Atchafalaya Basin, LA, USA, 2016, ORNL DAAC. 
Denbina, M., M. Simard, and J. Lai (2021), Pre-Delta-X: Lidar-derived Water Level Profiles in the Wax Lake Outlet, LA, USA, 2016, ORNL DAAC.

DHI (2021), MIKE 21 Flow Model FM, Hydrodynamic Module, User Guide, DHI Technologies.

Dietrich, J. C., S. Tanaka, J. J. Westerink, C. N. Dawson, R. Luettich, M. Zijlema, L. H. Holthuijsen, J. Smith, L. Westerink, and H. Westerink (2012), Performance of the unstructured-mesh, SWAN+ ADCIRC model in computing hurricane waves and surge, Journal of Scientific Computing, 52(2), 468-497, doi:10.1007/s10915-011-9555-6.

Douglas, D. H., and T. K. Peucker (1973), Algorithms for the reduction of the number of points required to represent a digitized line or its caricature, Cartographica: the international journal for geographic information and geovisualization, 10(2), 112-122, doi: 10.3138/FM57-6770-U75U-7727.

Edmonds, D. A., A. J. Chadwick, M. P. Lamb, J. Lorenzo-Trueba, A. B. Murray, W. Nardin, G. Salter, and J. B. Shaw (2021), Morphodynamic Modeling of RiverDominated Deltas: A Review and Future Perspectives, Earth and Space Science Open Archive ESSOAr, doi:10.1002/essoar.10507512.1.

Fagherazzi, S., M. L. Kirwan, S. M. Mudd, G. R. Guntenspergen, S. Temmerman, A. D’Alpaos, J. Van De Koppel, J. M. Rybczyk, E. Reyes, C. Craft, et al. (2012), Numerical models of salt marsh evolution: Ecological, geomorphic, and climatic factors, Reviews of Geophysics, 50(1), doi:10.1029/2011RG000359.

Ferziger, J. H., M. Perić, and R. L. Street (2002), Computational methods for fluid dynamics, vol. 3, Springer.

Große, F., K. Fennel, and A. Laurent (2019), Quantifying the relative importance of riverine and open-ocean nitrogen sources for hypoxia formation in the northern Gulf of Mexico, Journal of Geophysical Research: Oceans, 124(8), 5451-5467, doi:10.1029/ 2019JC015230.

Hagen, S., J. Westerink, R. Kolar, and O. Horstmann (2001), Two-dimensional, unstructured mesh generation for tidal models, International Journal for Numerical Methods in Fluids, 35(6), 669-686, doi:10.1002/1097-0363(20010330)35:6<669::AID-FLD108>3.0. $\mathrm{CO} ; 2-\% 23$.

Hiatt, M., and P. Passalacqua (2015), Hydrological connectivity in river deltas: The firstorder importance of channel-island exchange, Water Resources Research, 51(4), 22642282, doi:10.1002/2014WR016149. 
Hiatt, M., and P. Passalacqua (2017), What Controls the Transition from Confined to Unconfined Flow? Analysis of Hydraulics in a Coastal River Delta, Journal of Hydraulic Engineering, 143(6), doi:10.1061/(ASCE)HY.1943-7900.0001309.

Hiatt, M., E. Castañeda-Moya, R. Twilley, B. R. Hodges, and P. Passalacqua (2018), Channel-island connectivity affects water exposure time distributions in a coastal river delta, Water Resources Research, 54(0), doi:10.1002/2017WR021289.

Horritt, M., G. Di Baldassarre, P. Bates, and A. Brath (2007), Comparing the performance of a 2-D finite element and a 2-D finite volume model of floodplain inundation using airborne SAR imagery, Hydrological Processes: An International Journal, 21(20), 27452759, doi:10.1002/hyp.6486.

Horritt, M. S. (2000), Calibration of a two-dimensional finite element flood flow model using satellite radar imagery, Water Resources Research, 36(11), 3279-3291, doi:10. 1029/2000WR900206.

Huang, C., Y. Chen, S. Zhang, and J. Wu (2018), Detecting, extracting, and monitoring surface water from space using optical sensors: A review, Reviews of Geophysics, 56(2), 333-360, doi:10.1029/2018RG000598.

Isikdogan, F., A. Bovik, and P. Passalacqua (2017), RivaMap: An automated river analysis and mapping engine, Remote Sensing of Environment, 202, 88-97, doi:10.1016/j.rse. 2017.03.044

Jarriel, T., L. F. Isikdogan, A. Bovik, and P. Passalacqua (2020), System wide channel network analysis reveals hotspots of morphological change in anthropogenically modified regions of the Ganges Delta, Scientific Reports, 10(1), 1-12, doi:10.1038/ s41598-020-69688-3.

Jin, S., Y. Liu, S. Fagherazzi, H. Mi, G. Qiao, W. Xu, C. Sun, Y. Liu, B. Zhao, and C. G. Fichot (2021), River body extraction from sentinel-2A/B MSI images based on an adaptive multi-scale region growth method, Remote Sensing of Environment, 255, 112,297, doi:10.1016/j.rse.2021.112297.

Jones, C., M. Simard, and Y. Lou (2021), Pre-Delta-X: UAVSAR-derived Water Level Change Maps, Atchafalaya Basin, LA, USA, 2016, ORNL DAAC.

JPL (2021), The NASA Delta-X Project, https://deltax.jpl.nasa.gov/, accessed 2021-09-14.

Jung, H. C., M. Jasinski, J.-W. Kim, C. Shum, P. Bates, J. Neal, H. Lee, and D. Alsdorf (2012), Calibration of two-dimensional floodplain modeling in the central Atchafalaya 
Basin Floodway System using SAR interferometry, Water Resources Research, 48(7), doi:10.1029/2012WR011951.

Kim, B., B. F. Sanders, J. E. Schubert, and J. S. Famiglietti (2014), Mesh type tradeoffs in 2D hydrodynamic modeling of flooding with a Godunov-based flow solver, Advances in Water Resources, 68, 42-61, doi:10.1016/j.advwatres.2014.02.013Get.

LACPRA (2018), Coastwide Reference Monitoring System, Coastal Information Management System (CIMS) database, https://cims. coastal.louisiana.gov, accessed 2018-08-01.

Lane, S. N. (1998), Hydraulic modelling in hydrology and geomorphology: a review of high resolution approaches, Hydrological Processes, 12(8), 1131-1150, doi:10.1002/ (SICI)1099-1085(19980630)12:8<1131::AID-HYP611>3.0.CO;2-K.

Legrand, S., E. Deleersnijder, E. Hanert, V. Legat, and E. Wolanski (2006), Highresolution, unstructured meshes for hydrodynamic models of the Great Barrier Reef, Australia, Estuarine, coastal and shelf science, 68(1-2), 36-46, doi:10.1016/j.ecss.2005. 08.017

Leonardi, N., A. Canestrelli, T. Sun, and S. Fagherazzi (2013), Effect of tides on mouth bar morphology and hydrodynamics, Journal of Geophysical Research: Oceans, 118(9), 4169-4183, doi:10.1002/jgrc.20302.

Li, Z., and B. R. Hodges (2019), Modeling subgrid-scale topographic effects on shallow marsh hydrodynamics and salinity transport, Advances in Water Resources, 129, 1-15, doi:10.1016/j.advwatres.2019.05.004.

Liang, M., N. Geleynse, D. Edmonds, and P. Passalacqua (2015), A reduced-complexity model for river delta formation-part 2: Assessment of the flow routing scheme, Earth Surface Dynamics, 3(1), 87-104, doi:10.5194/esurf-3-87-2015.

Luettich, R. A., J. J. Westerink, N. W. Scheffner, et al. (1992), ADCIRC: An advanced three-dimensional circulation model for shelves, coasts, and estuaries. Report 1, Theory and methodology of ADCIRC-2DD1 and ADCIRC-3DL, Tech. rep., Coastal Engineering Research Center, U.S. Army Engineer Research and Development Center.

Marshak, C., M. Simard, M. Denbina, J. Nilsson, and T. Van der Stocken (2020), Orinoco: Retrieving a River Delta Network with the Fast Marching Method and Python, ISPRS International Journal of Geo-Information, 9(11), 658, doi:10.3390/ijgi9110658.

McFeeters, S. K. (1996), The use of the Normalized Difference Water Index (NDWI) in the delineation of open water features, International journal of remote sensing, 17(7), 
1425-1432, doi:10.1080/01431169608948714.

Mungkasi, S., and S. Roberts (2013), Validation of ANUGA hydraulic model using exact solutions to shallow water wave problems, in Journal of Physics: Conference Series, vol. 423, p. 012029, IOP Publishing, doi:10.1088/1742-6596/423/1/012029.

Musner, T., A. Bottacin-Busolin, M. Zaramella, and A. Marion (2014), A contaminant transport model for wetlands accounting for distinct residence time bimodality, Journal of hydrology, 515, 237-246, doi:10.1016/j.jhydrol.2014.04.043.

Nielsen, O., S. Roberts, D. Gray, A. McPherson, A. Hitchman, et al. (2005), Hydrodymamic modelling of coastal inundation, Modelling and Simulation Society of Australia and New Zealand Inc.

NOAA (2016), Tides and Currents, CO-OPS Center for Operational Oceanographic Products and Services, https://tidesandcurrents.noaa.gov/, accessed 2021-09-14.

Olliver, E., and D. Edmonds (2021), Hydrological connectivity controls magnitude and distribution of sediment deposition within the deltaic islands of Wax Lake Delta, LA, USA, Journal of Geophysical Research: Earth Surface, 126(9), e2021JF006,136, doi: 10.1029/2021JF006136.

Olliver, E. A., and D. A. Edmonds (2017), Defining the ecogeomorphic succession of land building for freshwater, intertidal wetlands in Wax Lake Delta, Louisiana, Estuarine, Coastal and Shelf Science, doi:10.1016/j.ecss.2017.06.009.

Otsu, N. (1979), A threshold selection method from gray-level histograms, IEEE transactions on systems, man, and cybernetics, 9(1), 62-66, doi:10.1109/TSMC.1979.4310076.

Paola, C., R. R. Twilley, D. A. Edmonds, W. Kim, D. Mohrig, G. Parker, E. Viparelli, and V. R. Voller (2011), Natural processes in delta restoration: Application to the Mississippi Delta, Annual Review of Marine Science, 3, 67-91, doi:10.1146/ annurev-marine-120709-142856.

Planet (2018), Planet Application Program Interface: In Space for Life on Earth, https: //api.planet.com.

PlanetLabs (2018), Planet imagery product specifications, Planet Team: San Francisco, CA, USA.

Roberts, H., J. Coleman, S. Bentley, and N. Walker (2003), An embryonic major delta lobe: A new generation of delta studies in the Atchafalaya-Wax Lake Delta system, Gulf Coast Association of Geological Societies Transactions, 53, 690-703. 
Roberts, K. J., W. J. Pringle, and J. J. Westerink (2019), OceanMesh2D 1.0: MATLABbased software for two-dimensional unstructured mesh generation in coastal ocean modeling, Geoscientific Model Development, 12(5), 1847-1868, doi:10.5194/ gmd-12-1847-2019.

Roberts, S., O. Nielsen, D. Gray, J. Sexton, and G. Davies (2015), ANUGA User Manual, Geoscience Australia.

Rouse, J. W., R. H. Haas, J. A. Schell, D. W. Deering, et al. (1974), Monitoring vegetation systems in the Great Plains with ERTS, NASA special publication, 351(1974), 309.

Schubert, J. E., and B. F. Sanders (2012), Building treatments for urban flood inundation models and implications for predictive skill and modeling efficiency, Advances in Water Resources, 41, 49-64, doi:10.1016/j.advwatres.2012.02.012.

Schumann, G., P. D. Bates, M. S. Horritt, P. Matgen, and F. Pappenberger (2009), Progress in integration of remote sensing-derived flood extent and stage data and hydraulic models, Reviews of Geophysics, 47(4), doi:10.1029/2008RG000274.

Shafiei, H., A. Soloy, I. Turki, M. Simard, N. Lecoq, and B. Laignel (2021), Numerical investigation of the effects of distributary bathymetry and roughness on tidal hydrodynamics of Wax Lake region under calm conditions, Estuarine, Coastal and Shelf Science, p. 107694, doi:10.1016/j.ecss.2021.107694.

Shaw, J., G. Kesserwani, J. Neal, P. Bates, and M. K. Sharifian (2021), LISFLOODFP 8.0: the new discontinuous Galerkin shallow-water solver for multi-core CPUs and GPUs, Geoscientific Model Development, 14(6), 3577-3602, doi:10.5194/ gmd-14-3577-2021.

Shaw, J. B., F. Ayoub, C. E. Jones, M. P. Lamb, B. Holt, R. W. Wagner, T. S. Coffey, J. A. Chadwick, and D. Mohrig (2016), Airborne radar imaging of subaqueous channel evolution in Wax Lake Delta, Louisiana, USA, Geophysical Research Letters, 43(10), 5035-5042, doi:10.1002/2016GL068770.

Simard, M., M. Denbina, D. Jensen, and R. Lane (2020), Pre-Delta-X: Water Levels across Wax Lake Outlet, Atchafalaya Basin, LA, USA, 2016, ORNL DAAC.

Siverd, C. G., S. C. Hagen, M. V. Bilskie, D. H. Braud, S. Gao, R. H. Peele, and R. R. Twilley (2019), Assessment of the temporal evolution of storm surge across coastal louisiana, Coastal Engineering, 150, 59-78, doi:10.1016/j.coastaleng.2019.04.010.

Swarzenski, C. M. (2003), Surface-water hydrology of the Gulf Intracoastal Waterway in south-central Louisiana, 1996-99, Tech. rep., US Department of the Interior, US Geolog- 
ical Survey.

Teng, J., A. J. Jakeman, J. Vaze, B. F. Croke, D. Dutta, and S. Kim (2017), Flood inundation modelling: A review of methods, recent advances and uncertainty analysis, Environmental modelling \& software, 90, 201-216, doi:10.1016/j.envsoft.2017.01.006.

Thomas, N., M. Simard, E. Castañeda-Moya, K. Byrd, L. Windham-Myers, A. Bevington, and R. R. Twilley (2019), High-resolution mapping of biomass and distribution of marsh and forested wetlands in southeastern coastal Louisiana, International Journal of Applied Earth Observation and Geoinformation, 80, 257-267, doi: 10.1016/j.jag.2019.03.013.

USGS (2016), U.S. Geological Survey National Water Information System, http: // waterdata.usgs.gov/nwis/, accessed 2021-09-14.

van der Walt, S., J. L. Schönberger, J. Nunez-Iglesias, F. Boulogne, J. D. Warner, N. Yager, E. Gouillart, T. Yu, and the scikit-image contributors (2014), scikit-image: image processing in Python, PeerJ, 2, e453, doi:10.7717/peerj.453.

Xing, F., J. Syvitski, A. Kettner, E. Meselhe, J. Atkinson, and A. Khadka (2017), Morphological responses of the Wax Lake Delta, Louisiana, to Hurricanes Rita, Elem Sci Anth, 5, doi:10.1525/elementa.125.

Yu, D., and S. N. Lane (2006), Urban fluvial flood modelling using a two-dimensional diffusion-wave treatment, part 1: mesh resolution effects, Hydrological Processes: An International Journal, 20(7), 1541-1565, doi:10.1002/hyp.5935.

Zhang, X., K. Xu, Z. Yang, X. Tan, and C. Wu (2021), Decreasing land growth and unique seasonal area fluctuations of two newborn Mississippi subdeltas, Geomorphology, 378, 107,617, doi:10.1016/j.geomorph.2021.107617. 